\title{
Bernhard Blanke Der deutsche Faschismus als Doppelstaat
}

Eine Auseinandersetzung mit: Ernst Fraenkel, Der Doppelstaat, Rückübersetzung aus dem Englischen von Manuela Schöps in Zusammenarbeit mit dem Verfasser, Frankfurt/Main-Köln 1974, 257 Seiten, Europäische Verlagsanstalt, 24,-DM*

Trotz umfangreicher Nationalsozialismusforschung zeitgeschichtlicher Provenienz stellen die Analysen der linken deutschen Emigration - der äußeren wie der inneren (als deren Produkt Fraenkel sein Buch bezeichnet, vgl. S. 13) - immer noch herausragende Arbeiten zum Faschismus dar. Erinnert sei an die Analysen von Franz Neumann, Otto Kirchheimer, Theodor W. Adorno und Max Horkheimer, Paul Sering, Arthur Rosenberg, schließlich an den 1973 zum ersten Mal publizierten Augenzeugenbericht von Alfred Sohn-Rethel'.

Diese Arbeiten wurden zum großen Teil erst in den späten 6oer Jahren in der Bundesrepublik bekannt, sei es, daß sie endlich übersetzt, oder - auch wenn sie immer noch nicht in deutscher Sprache vorliegen - durch die kritische, sich marxistischen Kategorien zuwendende Faschismus-Diskussion's sveröffentlichte wurden. In dieser Zeitschrift wurde vor allem die Funktion von Recht und Justiz im Nationalsozialismus diskutiert, wobei die zentralen Inhalte der Arbeit von Fraenkel wiederholt kritisch dargestellt oder zitiert wurdent. In dieser Untersuchung soll

* Zu Emse Fraenkel s. a. die Rezension in diesern Heft S. 326 von Wolfgang Luthardt. Zurn Prublem der Rolle der Justiz im Natıonalsozıalismus vgl. auch die Rezension von Richard Schmid in diesem Heft S. 33 t

' U. a. Franz Neumann, Behemoth. The Structure and Practice of Natzonal Soczalism, New York-London 1942 ('rg66), vgl. die Besprechung in KJ 1969, S. 317 f.; Otto Kirchhe1mer, Staatsgefüge und Recht des dritten Reiches (unter dem Pseudonym Dr. Hermann Seitz), in: Der deutsche Staat der Gegenwart, hg. Carl Schmitt, Hamburg r935; ders., Die Rechtsordnung des Natzonalsozialismus (194I), KJ 1971, S. 356 , auch abgedruckt in: ders., Funktionen des Staats und der Verfassung. 10 Analysen, Frankfurt/Main I 972 , S. 11 5-142; A. Gurland / Kirchhemer / Neumann, The Fate of Small Business in the Thurd Reich, Washington 1943; Theodor W. Adorno / Max Horkheumer, Dialektik der Aufklärung, New York 1944; Th. W. Adorno u. a., The Authontanan Personality, New York r950; Paul Senng, Die Wandlungen des Kapttalismus / Der Faschusmus, un: Zeitschrift für Soz1alismus, 2 (1935), Nr. 22-27; ders., Jensetts des Kaputalismus, Nürnberg 1946; Faschismus und Kapitalismus, hg. W Abendroch, Frankfurt 1967 (mit Arbeiten von O. Bauer, H. Marcuse, A. Rosenberg, A. Thalheimer); Alfred Sohn-Rethel, Okonomie und Klassenstruktur des deutschen Faschismus, hg. und einge!. von Agnoli/Blanke/Kadritzke, Frankfurt/ Main 1973.

- Neumanns Behemoth ist noch immer nicht in deutsch erschienen, ebenso emes der wichtigsten Bücher über die NS-Wirtschaft Zw1schen 1933 und 1937/38: Arthur Schwertzer, Big Business in the Thurd Retch, Bloomungton/USA 1964. Dagegen wurde das 1000 Seiten dicke Aufstieg und Fall des Drtten Retches von William L. Shirer (englisch 1960) innerhalb eines Jahres übersetzt und steht seit 196r auf den Bestsellerlisten. Die personalisıerenden Bücher über Hitler (Alan Bullock, Hitler, Düsseldorf ' 1967 und neuerdings Joachim C. Fests Hitler-Biographie) haben schnell die ro $\infty$ oer-Grenze überschntten.

, Vgl. die Arbeiten in den Zeitschriften Das Argument, Kritische Justiz, Probleme des Klassenkampfs.

- Vgl. insbesondere die Besprechung in Kritısche Justiz 1969, S. 319 f.; Helmut Ridder, Zur Verfassungsdoktrnn des NS-Staates, in: KJ 1969, S. 221-243; Alexander von Brünneck, Die Justız im deutschen 
deshalb versucht werden, das Werk in die allgemetne Faschismusdiskussion einzuordnen.

\section{Klassengesellschaft und demokratischer Verfassungsstaat - Zum Scheitern des Reformismus}

Allgemein gibt den Arbeiten der Emigranten die Tatsache ihre Bedeutung, daß sie von einem politischen "Standpunkt " aus geschrieben wurden, der keiner parteipolitischen Linie unmittelbar verpflichtet war. Entweder hatten die Autoren einen solchen Standpunkt nie eingenommen (vor allem die "Frankfurter Schule «) oder sie hatten sich im Verlauf der Krise der Weimarer Republik allmählich davon entfernt, sei es daß die politische Krise einen linkssozialistischen Kurs, wie ihn etwa Kirchheimer vertrat, völlig illusorisch erscheinen ließ, sei es, daß den sozialdemokratisch orientierten Autoren sukzessiv die Erkenntnis von der Unfähigkeit der deutschen Sozialdemokratie und der Gewerkschaften aufgedrängt wurde'. Fraenkel, der als Arbeitsrechtler, Anwalt und Syndikus den Gewerkschaften und der SPD verbunden war, entwickelte in diesem Prozeß eine scharfe Kritik des Reformismus der Weimarer Sozialdemokratie (vgl. S. I62 f., 207), die ihn zur Ausprägung marxistischer Argumentationslinien führte. Aber ähnlich wie anderen galt seine Parteilichkeit dem Proletariat als Klasse und nicht einem organisierten politischen Teil, bezog sich seine Kritik konsequent auf das Ganze der kapitalistischen Gesellschaft.

Diese Position stellte sowohl eine Stärke wie eine Schwäche dar. Ihre Stärke zeigt sich in der Analyse, weil der Faschismus aus der Sicht und im Interesse seiner Opfer analysiert wird. Sowohl Fraenkel als auch Neumann oder Kirchheimer haben in ihrer politzschen wissenschaftlichen Analyse des deutschen Faschismus das "Wesen" dieser bürgerlichen Herrschaftsform besser erkannt (und dabei eine bewundernswerte Materialfülle verarbeiter) als alle mit Quellenanalysen etc. gespickten zeitgeschichtlichen Werke nach ihnen. Die Schwäche ihrer Position (das gilt nicht für Kirchheimer) zeigt sich darin, daß die Rezeption des Marxismus ein Ergebnis der Enträuschung am Reformismus war, allerdings ohne jede Möglichkeit der praktischen »Umsetzung «. Dies war die Voraussetzung für die Relativierung des Marxismus zu einer bloßen "Methode» (so auch Schumacher nach 1945), die man später zugunsten anderer wissenschaftlicher und politischer Orientierungen und unter dem antikommunistischen Druck der bundesrepublikanischen Nachkriegszeit aufgeben konnte. Dieses Aufgeben des Marxismus war jedoch in doppelter Hinsicht ein Verdrängungs- und nicht ein Verarbeitungsprozeß. Zum einen war schon die Rezeption des Marxismus ein Produkt der Krise der bürgerlichen Gesellschaft seit dem Ende der zoer Jahre, welches den krisenerzeugten Erscheinungsformen des Kapitalismus verhaftet blieb und dessen Grundstrukturen nicht zureichend erfaßte. Zum anderen holte der "Schock " des Stalinismus die alten reformistischen Vorstellungen wieder aus der Versenkung, je mehr Marxismus und Stalinismus identifiziert wurden.

In seinen biographischen Selbstdarstellungen kommt dies bei Fraenkel deutlich zu Tage. Entweder er entschuldigt sich für seine "an mehr als einer Stelle ein wenig ,vulgär-marxistisch« erscheinenden Schriften damit, daß am Ende der Weimarer Zeit die Philosophisch-Okonomischen Manuskripte noch nicht bekannt gewesen

Faschismus, in: KJ 1970, S. 2 I-35; s. a. die werteren Nachweise in KJ 1970, S. 1, Fn. 50wie in den jährlichen Inhaltsverzeichnissen der KJ unter den Stıchworten *Nationalsozıalismus und Recht « und -Rechtsgeschichteж; s. a. die in diesem Heft S. 331 in der Fußnote zıtıerten Rezensionen der KJ.

, Vgl. Neumann, Behemoth, S. 34 f., 45. 
seien ${ }^{\dagger}$ oder er relativiert seine Neigung, „die Richtigkeit der marxistischen Analyse des Faschismus anzuerkennen * mit der Bemerkung, dies sei nur möglich gewesen, weil die "fundamentalen Veränderungen, die sich in der UdSSR unter dem Regime Stalins und in den USA im Zeichen des New Deal vollzogen « hätten, von ihm damals völlig vernachlässigt worden seien. Dieser Hinweis auf die »nicht einheitliche" Einstellung zum Marxismus, die Fraenkels "gesamtpolitische Entwicklung "? reflektiert, soll nicht ein Hinweis auf etwaiges "Renegatentum« sein, wie er von links oft gegen ehemalige Marxisten vorgebracht wird. Es geht um die Brüche in seiner theoretischen Entwicklung, die von seinen Anhängern gern geglättet werden. Fraenkel selbst hat, wenn auch zögernd, seine "marxistischen « Schriften wieder veröffentlicht und ihren analytischen Wert, den sie seinem radikalen Engagement für die Arbeiterklasse und der Rezeption der damals bekannten Marxschen Werke verdanken, weiterhin anerkannt.

Durch die späte Veröffentlichung ist allerdings jene Spurenverwischung begünstigt worden, der die Erkenntnis des Zusammenhangs von Kapitalismus und Faschismus jahrelang zum Opfer gefallen ist. Und es ist bezeichnend, daß die Gralshüter der westdeutschen Nachkriegsideologie in Fraenkel im wesentlichen nur den Theoretiker des »Neopluralismus « sehen ${ }^{8}$ und die Spurenverwischung auch auf den Doppelstaat « ausdehnen möchten. Das hat natürlich seine Gründe. Der "Doppelstaat" stellt eine Provokation in mehrerer Hinsicht dar: er widerlegt erstens die nach 1945 kunstvoll - vornehmlich aus legitimatorischen Gründen ausgebaute Totalitarismustheorie schon zu einem frühen Zeitpunkt (vgl. z. B. S. 2 I, 92 f., 1 30) derart präzise, $\mathrm{da} ß$ eine frühere Veröffentlichung in der BRD objektiv nicht »in die Landschaft « paßte. So galt und gilt das Buch den Zeitgeschichtlern und Politologen als Buch der "Erfahrungen mit dem Totalitarismus " , dem zugleich entgegengehalten wird, durch seine Beschränkung auf eine bestimmte Zeitspanne (1933-38) das "Wesen" des totalitären Regimes nucht voll erkannt zu haben'. Zweitens analysiert Fraenkel Phänomene der justizförmigen Verfolgung politischer Gegner und des politischen Ausnahmezustandes in einer bürgerlichen Gesellschaft, die keineswegs auf den Nationalsozialismus beschränkt sind (vgl. zur historischen Kontinuität S. 184-202), sondern - etwa in der Kommunistenverfolgung in den soer und Anfang der 6oer

${ }^{6}$ Ennst Fraenkel, Zur Sozwlogıe der Klassenjussız und Aufsätze zur Verfassungskrise 1931 1-32, mit einem Vorwort zum Neudruck, Darmstadi 1968 , S. XI.

' Ernst Fraenkel, Reformismus und Pluralismus. Materialien zu einer ungeschriebenen politischen Autobrographre, zusgest. und hg. von Falk Esche und Frank Grube, Hamburg 1973, S. 25 f. (Vgl. die Besprechung in diesem Heft S. 326).

${ }^{8} \mathrm{Vgl}$. die Beiträge von Schwan, Steffani und Kremendahl in Günther Doeker / Winfried Steffani, Klassenustiz und Pluralismus. Festschrift für Enst Fraenkel zum 75. Geburtstag, Hamburg 1973.

9 Hans Kremendahl, Von der dialektuschen Demokratze zum Pluralismus. Kontınutät und Wandel ım Werk Ernst Fraenkels, in: Doeker/Steffanı (FN 8), S. 381-394, $3^{86 .}$

to Dieser Einwand wird sowohl von seiten der Totalitansmustheorte vorgebracht wie von seiten an einer Theone des Faschismus als Herrschaftsform des Monopolkapitals orientierten Autoren. Hans Buchheim (Die SS - Das Herschaftsinstrument, in: Buchherm/Broszat/Jacobsen/Krausnick, Anatomie des SS-Staates. Gutachten des Instituts für Zettgeschichte zum Ausschwitz-Prozeß, Bd. I, Olten und Freiburg 1965, S. 2 ff.) setzt der Fraenkelschen These vom Doppelstaat die These von einem Nebeneinander waußernormatıver Führergewalt * und *normatıver Staatlichkeıt *ntgegen, welches schrittweise dadurch aufgehoben wurde, daß die $*$ Führergewalt « die -Staatlichkeıt « schließlich verdrängt habe. Dies resultuert aus der Logik der Terronnstrumente, der Buchheim folgt und bei deren Verfolgung er die nichtterrorist1schen Reproduktions- und Herrschaftsformen des Faschismus außer acht läßt (vgl. hierzu Punkt III dieser Arbeit). Auch von der anderen Seite wird Fraenkels These kntusiert, weil sie die terrorstische Form der Herrschaft des Kapitalismus unterschätze. Vgl. die Besprechung des Buches von Franciszek Ryszka, Der Staat des Ausnahmezustandes (poinisch) in KJ I970, S. I 6 f. Reinhard Opıtz (Ober die Entstehung und Verbinderung von Faschismus, in: Das Argument, I6 [1974], Nr. 87, S. 543-603) geht so weit, Fraenkel in die Reihe der Rehabilitanten des Faschısmus eınzureihen, die über die Konstatıerung einer - pluralisuschen $\alpha$ Herrschaftsform des Faschismus diesen vom Vorwurf des Totalitären fressprechen, um itin nun voll gegen den Kommunısmus zu wenden ( $\mathrm{S}$. $547 \mathrm{ff}$.). So recht Opitz in der Kritik der Revision der Totalitarısmustheorie und ihrer Motivation haben mag, Fraenkel trifft dieser absurde Vorwurf nicht. 
Jahre sowie in den Berufsverboten seit 1971 - auch ansatzweise in der Bundesrepublik möglich waren und sind. Und drittens beschreibt der »Doppeistaat*, wenn man ihn im Zusammenhang mit den Arbeiten zur Klassenjustiz und zur Verfassungskrise der Weimarer Republik sieht, das Scheitern reformistischer und liberal-demokratischer Illusionen angesichts einer globalen Faschisierung nicht in Termini der Resignation und der Anpassung, sondern in Begriffen eines politischen und intellektuellen Widerstandes, der beispielhaft wirken könnte.

Als Anwalt der Gewerkschaftsbewegung hoffe Fraenkel vor dem Faschismus ebenso wie seine "Klienten«, dem Kapitalismus im Rahmen bürgerlich-demokratischer Verfassungsinstitutionen Stück für Stück Rechtspositionen abringen zu können, die sowohl im individuellen und kollektiven Arbeitsrecht als auch in der allgemeinen Gesetzgebung Bestandteil einer »unverbrüchlich geltenden Rechtsordnung «" werden sollten, hinter die es kein Zurück mehr geben konnte. Er ging dabei, wie viele linke Theoretiker in der Weimarer Zeit, von dem schon von Marx konstatierten allgemeinen Widerspruch aller bürgerlichen Verfassungen aus, nach dem die beherrschten Klassen, vor allem die organisierten Lohnarbeiter durch das allgemeine Wahlrecht in den Besitz der politischen (ohne die gesellschaftliche) Macht gesetzr werden, daß aber die herrschende Klasse in ihrer gesellschaftlichen Macht ohne eindeutige politische Garantien bestätigt würde ${ }^{11}$. Mittels der Legislative sollte der organisierte Kampf der Arbeiterbewegung die nur parlamentarische Demokratie schrittweise gesellschaftlich fundieren und zur »sozialen Demokratie« oder wie Fraenkel es ausdrückte: zur »kollektiven« oder »dialektischen “ Demokratie weiterführen's. Diese Vorstellungen gingen sowohl von der Erkenntnis des Klassencharakters der Weimarer Gesellschaft und damit von der Labilität des politischen Zustandes aus, wie sie zugleich meinten, auf der Basis der Parität, des politischen und - bezogen auf den Verteilungskampf - ökonomischen Gleichgewichts der Klassen durch eine "Vergesetzlichung der Wirtschaft " die Vorherrschaft der bürgerlichen Klasse brechen, diese gar »aus dem Eigentum . . . verdrängen "' zu können. Der Widerspruch zwischen Klassengesellschafr und demokratischem Verfassungsstaat sollte einmal durch die Integration der Organisationen der Arbeiterbewegung in den Staat und durch politische Kompromisse mit den gesellschaftlich Herrschenden aufgehoben werden. Zum andern wies die Konzeption der Uberwindung der Klassenstruktur über dieses Kompromißsystem hinaus, hielt der Reformismus am Endziel, dem Sozialismus fest. Die Konzeprion Integration der Arbeiterbewegung in den demokratischen Staat beinhaltete die Vorstellung von dessen Neutralität, während in der Konstatierung des Klassencharakters der Gesellschaft die Erkenntnis des Klassencharakters des Staates angelegt war. In den Analysen zur Klassenjustiz arbeitet Fraenkel diesen Charakter heraus. In den Schriften zur Verfassungskrise 1931-1932 wird für ihn zunehmend deutlich, wie die Weimarer Verfassungskompromisse und deren gesellschaftlich-politische Fundierungen (die "Arbeitsgemeinschaft «) von der herrschenden Klasse aufgekündigt wurden. Die

1" Vgl. die Argumentation in Sozıologıe der Klassenjustzz. . (FN 6) und im Doppelstaat, S. $136 \mathrm{ff} .$, I 52 ff., 160.

'a Kari Marx, Die Klassenkämpfe in Frankreach 1848-1850, in: Marx-Engels-Werke, Berlin (DDR), Bd. 7, S. 43. Dieser Widerspruch spielte vor allem in den Verfassungsanalysen Otto Kirchheumers eune große Rolle. Vgl. erwa ders., Weimar - und was dann? Analyse esner Verfassung (1930), in: ders., Politik und Verfassung, Frankfurt/Maun 1964, S. 9-56.

is Vgl. hierzu Bernhard Blanke, Theonen zum Verhältnis von Staat und Gesellschaft, Kapitel 7.4., in Blanke/Jürgens/Kastendiek, Kritik der Politischen Wissenschaft, 2 Bde., FrankfurdMann-New York 1975, S. $162-168$.

14 Hermann Heller, Rechtsstaat oder Diktatur (1929), in: ders., Gesammelte Schriften, hg. von Draht u. a., 3 Bde., Leiden 197I, Bd. II, S. $46 \mathrm{r}$

is A. a. O., S. 448. 
Ambivalenz der politischen Zustände wird zugunsten der gesellschaftlichen Machtstruktur entschieden.

In dieser Zeit hatte Fraenkel, ebenso wie Hermann Heller, die Hoffnung, daß über wegen der großen Krise offensich tlich notwendige ${ }^{16}$ - Veränderungen des politischen Systems wenigstens der Rechtsstaat gerettet werden könnte. Als das nicht mehr möglich schien, zog er - im Gegensatz zu Heller - revolutionäre Konsequenzen: nicht mehr die Parität sei das Ziel, sondern die

^Herrschaft der Arbeiterklasse ... Zur Erreschung dieses Ziels bedarf unter den gegenwärtigen Verhältnissen die Arbeiterschaft der Freiheitsrechte und der demokratisch-politischen Befugnisse, die die Weimarer Verfassung ihr gewährleistet hat. Wenn das Proletarıat für diese Rechte kämpft, geht es den geraden Weg zu seiner sozialen und wirtschaftlichen Befreiung “"

Nachdem die SPD den Abbau der demokratisch-politischen Befugnisse mit der Tolerierungspolitik geduldet hatte, war es 1932 für diesen Kampf schon zu spät. In seiner Tätigkeit als Anwalt, der die Entstehung des "Doppelstaat* zu verdanken ist, mußte Fraenkel nun nach 1933 erfahren, daß der Rückfall gegenüber Weimar nicht nur die Revozierung erkämpfter kollektiver und individueller Rechte der Arbeiterklasse mit sich brachte, sondern daß mit dem Umbau des politischen Systems, mit der Abschaffung des Pariamentarismus zum Zwecke der Krisenlösung der Staat, dessen $»$ Benutzung in Weimar so viele Hoffnungen gegolten hatten, seiner liberaldemokratischen Qualität entkleidet und als Klassenstaat rekonstruiert wurde. Aber diese Rekonstruktion machte vor den Klassengrenzen nicht hait. Alle Staatsbürger wurden einer Freund-Feind-Einteilung unterzogen, die (potentiell) über politwsche Gegnerschaft hinaus im "Rassestaat « die physische Existenz willkürlich ausgebürgerter Gruppen bedrohte und schließlich vernichtete.

\section{Der Doppelstaat als Resultat faschistzscher Feindbekämpfung}

Diese allgemeine Feind-Bekämpfung - zu Beginn der NS-Herrschaft scheinbar nur auf den Kommunismus konzentriert, dann aber mittels der Theorie und Praxis der Bekämpfung einer »mittelbaren kommunistischen Gefahr « $(S .43 \text { f. })^{18}$ diffus ausgeweitet - zerstörte schrittweise den allgemeinen rechtlichen Rahmen der bürgerlichen Gesellschaft, den Rechtsstaat. Im Hauptteil seines - im übrigen hervorragend übersetzten - Buches (Die Rechtsordnung des Doppelstaates, S. 25-134) analysiert Fraenkel anhand einer Rechtssprechungsanalyse bis 1938 das Heraustreten einer wie er es nennt - »politischen Gewalt« und einer »politischen Sphäre o ohne jegliche Rechtsgarantien aus der unter der Weimarer Verfassung und dem Rechtsstaat geitenden Rechtsordnung. Dieser spezifische »politische Sektor* (S. 26, 98) und diese "politische Gewalt" gründeten sich auf den durch die Notverordnung zum Schutz von Volk und Staat vom 28. Februar 1933 in Permanenz gesetzten Belagerungszustand (S. 26 ff.). Sie stützten sich in ihrer "Legitimität« auf die - durch das Ermächtigungsgesetz in scheinlegale Bahnen gelenkte - Diktatur des Führers und Reichskanzlers Hitler, sie wurden exekutiert von den diversen Machtträgern in Staat und Partei. Auf diese "politische Gewalt « gründete sich der Maßnabmenstaat, der

is Vgl. den Aufsacz Verfassungsreform und Sozıaldemokratie (1932), in: Zur Sozıologie der Klassenjustzz . (FN 6), S. 89-103.

"Ernst Fraenkel, Abschied von Wesmar'(1932), a, a. O., S. $57-72,72$.

13 So stellte das Landgenchs Berlin in emem Urteil vom 1. 1 1. 1933 in Anwendung der Notverordnung vom 28. 2. fest: -Alle gegen die öffentliche Sicherheit und Ordnung ... genchteten Angriffe (werden) als kommunıstısch im weıtesten Sinne aufzufassen seın * (zıtiert nach Fraenkel, S. 45 ). Wem fällt da nucht die *Sympathisanten $\alpha-$ oder $*$ Dunstkre1s $\alpha$-Theorte neuesten Datums esn? Oder die $*$ Kontaktschuld im soer-Jahre-Strafrecht? 
alles geltende Recht (Legalität) unter den »Vorbehalt des Politischen« stellte, d. h. sowohl unter den Vorbehalt der politischen Ideologie und Zwecke des Nationalsozialismus wie unter die faktische Verfügung des staatlichen Gewaltapparates (Polizei, später SS). Es gab - schreibt Fraenkel -

»keine Materie des gesellschaftlichen oder wirtschaftlichen Lebens, die dem Zugriff des Maßnahmenstaates entzogen ist (S. 73) ... Die gesamte Rechtsordnung steht zur Disposition der politischen Instanzen. Sowert jedoch die politischen Instanzen von ihren Machtbefugnissen keınen Gebrauch machen, regelt sıch das private und öffentliche Leben nach den Normen des überkommenen oder neugeschaffenen Rechts $\propto$ (S. 88).

Die Frage nach dem gesellschaftlichen Inhalt des „Politischen «, nach den strukturellen Grenzen der Feindbekämpfung beantwortet Fraenkel mit dem Nachweis der Existenz des Normenstaates. Theoretisch ist »die Zuständigkeit des Maßnahmenstaates unbeschränkt, faktisch jedoch beschränkt. Dies ist das wichtigste Kriterium der gegenwärtigen Verfassung Deutschlands « (S. 89). Denn der Nationalsozialismus hat nicht nur Feinde, er kennt und anerkennt auch die "positiv aufbauenden Kräfte des Volkes « und diese »stehen grundsätzlich unter dem Schutz des Normenstaates (S. 94/95). Den positiv aufbauenden Kräften gegenüber kann der Staat auf die Normierung und Berechenbarkeit seines Verhaltens nicht verzichten, ihnen müssen Rechtsgarantien gewährt werden. Werden einerseits also im Maßnahmenstaat die Akte der Gestapo (und damit der SS) dem geltenden Rechtsstaat und der Nachprüfung durch die Gerichte entzogen (und zwar durch Anerkennung der Gerichte selbst! ( $($. 54 f., 69)), so werden andererseits durch den Normenstaat das Privateigentum und seine Konnexinstitute weiterhin geschürzt (bis 1938 auch das jüdische Privateigentum) (S. 104-127).

Im Begriff des "Doppelstaates kommt bei Fraenkel zweierlei zum Ausdruck:

I. Der Normenstaat ist gewissermaßen ein halbıerter »Rechtsstaat«. Denn unter Rechtsstaat versteht Fraenkel (im Gegensatz etwa zu Carl Schmitt's) die Einheit von öffentlichen, politischen Rechten und privaten, unpolitischen Rechten, d. h. die Einheit von demokratischem Verfassungsstaat und universaler Geltung rechtsstaatlicher Prinzipien. Im Normenstaat des Nationalsozialismus sind nur noch die kapitalfunktionalen Normen des Privatrechts und solche öffentlichen Rechte, die sie unabdingbar nach sich ziehen (z. B. im Verkehr der Privateigentümer mit der Verwaltung oder im Steuerrecht), in Kraft, während der größte Teil der Bevölkerung von der Bestimmung der politischen Gewalt ausgeschlossen, einer potentiellen Verfolgung unterworfen und in seinen privaten Rechten, weil er nicht über Privateigentum an "Sachmitteln« verfügt, fast völlig beschnitten ist. Während also der Rechtsstaat, der ungeteilt für alle gilt, Element des demokratischen Verfassungsstaates ist, ist der Normenstaat, der auf die Privateigentümer an »Sachmitteln * begrenzt ist, Element des faschistischen Klassenstaates.

2. Im Zusammenspiel von Aufrechterhaltung der kapitalistischen $>$ Grundordnung، und Feindbekämpfung ergibt sich die notwendige Symbiose von Normenstaat und Maßnahmenstaat. Die von den normativen und außernormativen Möglichkeiten her konstatierte Konkurrenz zweier »Herrschaftssysteme - wie Fraenkel wiederholt den Maßnahmenstaat und den Normenstaat nennt (S. 21, 75) - erweist sich als von der gesellschaftlichen Wirklichkeit her strukturell vorbestimmt. Zeigt somit Fraenkel an der Rechtsordnung (d. h. den Normen und Verfahrensweisen) im einzelnen die Durchsetzung der faschistischen Herrschaft und deren Begrenzung durch das geltende, für die Reproduktion einer bürgertichen Gesellschaft, notwendige Recht, so liegt doch genau in der Differenz von ntotalitären « Möglichkeiten und begrenzter

19. Vgl. Carl Schmitt, Verfassungslehre, Berlin 1928, S. 125, $200 \mathrm{ff}$. 
Wirklichkeit des Maßnahmenstaates die Hauptschwierigkeit für das Verständnis des Buches. Auf der Ebene der Rechtsnormen und der juristischen Verfahrensweisen nämlich sind die "polirische Gewalt« und der "politische Sekror* bei Fraenkel zunächst nur als außerhalb der überkommenen Rechtsordnung stehend definiert. So erscheinen sie als außerhalb der kapitalistischen Gesellschaft stehend, dieser gegenüber nur durch die Tolerierung bestimmter Normen und Verfahrensweisen begrenzt. Die - an der positivistischen Methodik orientierte - Trennung der Untersuchung von Rechtsordnung und Rechtswirklichkeit bei ihm kann deshalb als Auseinanderreißen des funktionellen Zusammenhangs mißverstanden werden. Tarsächlich aber versucht Fraenkel gerade, die "politische Gewalt* historisch-soziologisch (Kapitel III) als Funktion dieser Gesellschaft zu bestimmen. Die analytische Trennung bedingt einen Wechsel der Betrachtungsebenen. Während in der Untersuchung der Zerstörung des Rechrsstaates zunächst einmal dieser in seiner relativ eigenständigen Struktur vorausgesetzt wird, wird bei der historisch-soziologischen Identifizierung der "politischen Gewalt« der Zusammenhang von kapitalistischer Gesellschaft und Staat thematisiert.

Im Abschnitt zur Rechtsgeschichte eines Doppelstaates in Preußen und Deutschland (S. 1 84-202) analysiert Fraenkel die fehlgeschlagenen Versuche, einen bürgerlich-liberalen Verfassungsstaat im Einklang mit der kapitalistischen Entwicklung in Deutschland zu etablieren. Beschrieben werden die Kompromisse zwischen "herrschender Klasse « und »monistischer Staatsgewalt* von der Begründung der absoluten Monarchie bis zum Rechtsstaat der Weimarer Republik. Problematisch in diesem Versuch, den Faschismus in die deutsche Tradition einzuordnen, ist die Abstraktion, die Fraenkel hier am Begriff der »politischen Gewalt« vollziehr. Ihm geht es um den Nachvollzug der Genese und Existenz jener »spezifischen (S. 20I) Gewalt, die unterhalb und jenseits aller rechtsstaatlichen Bindungen existiert, im Dienste der herrschenden Klasse eingesetzt wurde und wird, aber nicht in ihrer Verfügung steht.

"Soziologisch ist für den Doppelstaat die Tatsache kennzeichnend, daß die herrschende Klasse berett ist, unter folgenden Bedingungen in die Errichtung einer monistischen Staatsgewalt eınzuwilligen:

I. daß die für ihre ökonomische Existenz lebenswichtigen Vorgänge nach einem ihr tragbar erschernenden Recht geregelt werden;

2. daß die beherrschte Klasse ökonomisch entmachtet wird, nachdem ihr vorher als kollektive Einhert der Schurz der Rechtsordnung entzogen worden ist* (S. 185).

Diese Definition erinnert an die aus der Bonapartismustheorie stammende Problematik der »Verselbständigung der Exekutivgewalt « ${ }^{20}$. Im Rahmen dieser Diskussion wird aber eine solche Verselbständigung als Resultat einer Krise der bürgerlichen Gesellschaft angesehen, während Fraenkel ja gerade versucht, das spezifisch deutsche Problem der nicht statrgehabten bürgerlichen Revolution und der nicht vollzogenen Unterwerfung der staatlichen "Souveränitär* unter die bürgerliche Klasse zu begreifen.

Für den demokratischen Verfassungsstaat der Weimarer Requblik steilte sich das Problem dann auch anders:

"Die Revolution von 1918 hatte zwar den formalen Dualismus des Staates beseitigt, nicht aber den politischen Einfluß jener imperıalistisch-großkapitalistisch-protektionisuschen Kreise, die seıt der Bismarckzeit Befürworter der Machtpolitik gewesen waren* (S. 20I).

20 Ausgehend von den Marxschen Schriften zur Entwicklung der Klassenkämpfe in Frankresch nach 1848 und zum Bonapartismus hat Ende der zoer Jahre vor allem August Thalheimer versucht, den Faschismus in diesem theoretıschen Kontext zu erklären. Vgl. hierzu Volker Kröning / Jakob Schissler, Die Exekutivgewalt in der Bonapartismus- and Faschismustheone, in: KJ 1970, S. 2-9; Niels Kadritzke, Faschismus als gesellschaftliche Realität und als unrealistischer Kampfbegriff, in: Probleme des Klassenkampfs, Nr. 8/9, 1973 , S. 103-143. 
Die Staatsgewalt selbst war nicht mehr geteilt in monarchische Exekutive und bürgerliche Gesetzgebung, es gab verfassungsrechtlich keine außerhalb der parlamentarischen Gesetzgebung und Kontrolle existierende reine "politische Gewalt* mehr. Die Verfechter der Machtpolitik (womit Fraenkel die Koalition von Schwerindustrie und Großagrariern meint) strebten jedoch danach, sie wieder zu errichten, und zwar mit den Alternativen,

wentweder (a) praeter legem eine außerhalb der rechtsstaatlichen Ordnung stehende politische Gewalt zu etablieren und die Verfassung mit dem Ziel der Begründung eines autoritären Machtstaates zu revidieren, oder (b) contra legem eine Diktatur an die Stelle der rationalen rechtsstaatlichen Verfassungsordnung zu setzen* (S. 20I).

Die erste Alternative sieht er in der Ära Brüning verwirklicht, mit dem Nebeneinander von außerordentlicher Präsidialgewalt aufgrund Art. 48 WRV einerseits und der Aufrechterhaltung eines beträchtlichen Teils der rechtsstaatlichen Ordnung andererseits. Die zweite Alternative war der Nationalsozialismus.

Fraenkel übersieht bei dieser Ahnenreihe, daß bereits im Kaiserreich eine Funktionsveränderung des Staates stattgefunden hatte. Selbst wenn verfassungsrechtlich und organisatorisch die traditionellen Gewalten der konstiturionellen Monarchie im Gegensatz zum bürgerlichen Parlament gestanden haben, so wurde der Staat mit der forcierten Entwicklung des deutschen Kapitalismus und zumal im ersten großen imperialistischen Krieg funktionell ein bürgerlicher Staat ${ }^{21}$. Die Weimarer Verfassung hat, über die von Fraenkel u. a. konstatierte Tatsache hinaus, daß die Träger der Staatsgewalt die aiten waren (preußische Bürokratie und Justiz, Reichswehr mit ihrem Sonderstatus), an dieser funktionellen Einbindung des Staates in die kapitalisrische Gesellschaft prinzipiell nichts verändert. Ein "Doppelstaat « drohte nun von einer anderen Seite. Die Weimarer Verfassung stand gewissermaßen "links« von der Gesellschaft und vom alten Staatsapparat ${ }^{22}$. Unter ihrer Geltung drohten auf eine Massenbasis sich stützende parlamentarische Mehrheiten den Staat als Mittel gesellschaftlicher Veränderungen zu ge- oder (aus der Sicht der herrschenden Klasse) mißbrauchen. Ein funkrionell durch seine Beziehungen zur kapitalistischen Gesellschaft geprägter Klassenstaat war in eine politisch ambivalente, weil demokratische Verfassung eingebunden. Mit der Machtergreifung des Faschismus trat nun einerseits die Staatsgewalt aus diesen demokratisch-rechtsstaatlichen Bindungen hervor, andererseits stellte sich für die herrschende Klasse das Problem der politischen Garantie für die adäquate Funktionsweise des Staates anders. Es galt nun eine faschistische Massenbewegung, die nicht originär, wie ehemals der Feudaladel, sondern auf dem - wie immer illegalen - Weg über den Parlamentarismus an die Macht gelangt war, daran zu hindern, den Staat funktionell anders als im bürgerlichkapitalistischen Sinne zu verwenden.

Der spezifisch »politische (d. h. nicht rechtlich rational eingebundene) Einsatz der Staatsgewalt, wie er sich in der Permanenz des Belagerungszustandes etablierte, bestand auch nicht mehr in der Verfügung über das Militär und die Außenpolitik allein, wofür die überkommenen Gewalten der konstitutionellen Monarchie noch ihre traditionale, jedoch in rechtliche Formen einbindbare Legitimation besaßen. Das "Politische " bestand vielmehr in der an die Aufrechterhaltung der kapitalistischen Gesellschaft gebundenen abstrakten Funktion der allumfassenden Feindbe-

"Vgl. huerzu Joachim Hirsch, Wissenschaftlich-technuscher Fortschrtt und politzsches System, Frankfurt/ Main 1970, S. I1-40; Peter von Oerzzen, Die soztale Funktion des staatsrechulichen Posttivismus, Frankfurt/Main 1974 .

"Ridder (FN 4), S. 231 . Vgl, auch die Untersuchung von Joachim Perels, Kaptalismus und politische Demokratte. Prvatrechtssystem und Gesellschaftsstruktur in der Wetmarer Republik, Frankfurt/Main 1973 . 
kämpfung. Der faschistische Begriff des "Politischen « hat in seiner schon von Carl Schmitt vordefinierten Bedeutung ${ }^{23}$ den Inhalt der „Objektlosigkeit«. Diese Objektlosigkeit der innen- und außenpolitischen Feindbekämpfung ${ }^{4-}$ mach Irrationalität der faschistischen "Weltanschauung * als Versuch der Legitimitätsbegründung des politischen Herrschaftssystems aus (vgl. S. 233-24I).

Bezieht man jedoch die Freund-Feind-Bestimmungen funktionell auf die bürgerlich-kapitalistische Gesellschaft zurück, resultiert aus ihr notwendig jener Doppelstaat, den Fraenkel spezifisch für den Nationalsozialismus analysiert hat. Hier trifft dann auch seine allgemeine und zentrale Definition zu:

"Von einem Doppelstaat sollte nur dann gesprochen werden, wenn die Staatsgewalt strukturell enheitlich organisiert ist, ihre Handhabung jedoch funktsonell nach verschiedenen Methoden in Erscheinung tritrk (S. I8 5 , Unterstr. v. u.).

Der Faschismus hat die Staatsgewalt des deutschen Reiches insofern unter einer Zentrale »einheitlich organisiert «, als die diversen, durch den Verfassungsstaat der WRV gesetzten Gewaltenteilungen zwischen Parlament und Exekutive, Reichsgewalt und Länderhoheit abgeschafft wurden. Mit der Außerkraftsetzung der Grundrechte wurden darüberhinaus die Grenzen zwischen "Staat« und "Gesellschaft» formell beseitigt.

Die traditionelle Totalitarismustheorie, die sich allein auf die Untersuchung des politischen Herrschaftssystems beschränkte, ging von diesem Befund aus und konstatierte eine durchgehende "Politisierung" der Gesellschaft, d. h. eben den "totalen « Staat"s. Fraenkel weist demgegenüber in seinem Buch schon früh darauf hin, daß dieser Staat - in den Worten von Carl Schmitr - nicht quantitativ, sondern "qualitativ * total war ${ }^{26}$. Als bürgerlicher Staat mußte der Herrschaftsapparat des NS die Grenzen zwischen der zentralen staatlichen Gewalt und der Souveränität des Kapitals über den gesellschaftlichen Reproduktionsprozeß weiterhin achten, im Gegensatz zur Weimarer Republik überhaupt erst wieder herstellen. Einer »Politisierung* der kapitalistischen Gesellschaft waren somit von vornherein konkrete, »objekthaltige« Grenzen gesetzt. Die von Carl Schmitt nach 1933 entwickelte Theorie vom »konkreten Ordnungsdenken «" war ein Versuch, die Ausuferung des feindbestimmten "Politischen « einzudämmen und ihm eine Richtung zu geben, die darin bestand, die durch das Privateigentum gesetzten wunpolitischen* Herrschaftsbereiche in Gestalt der "Stände" aus dem unmittelbaren Verfügungsbereich des Staates auszugliedern. Diese "Stände« waren nichts anderes als die Bereiche gesell-

"Carl Schmitt, Der Begriff des Polituschen, Berlin 1927.

24 Hierfür stehen exemplarısch die sıch nach 1933 immer weiter ausdehnenden geseczlichen Feinddefinutınen im Faschismus. Auf die Kommunisten folgten die Juden (Nürnberger Gesetze), dann die Gewohnheirsverbrecher, die >Asozialen *, die Polen, die Russen etc. Vg!. Martzn Broszar, Natzonalsoz zalistzsche Konzentrattonslager 1933-1945, in: Buchheim u. a., Anatomie des SS-Staates (FN 10), S. 9-160.

1s Trotz aller mittlerweile effolgren Modifizierungen in dieser Theorie kreist ihre zentrale Argumentation immer noch um die totalitäre Konzentration aller polituschen Machtmittel (Staat, Polizes, Geheımdienst, Massenmedien, Militär) und die völlige Unterwerfung aller autonomen gesellschaftlichen Interessenorganısationen oder politischen Arrikulationen. Die Grundmuster dieser Theorıe haben ausgearbestet: Hannah Arendr, Elemente und Ursprïnge totaler Herrschaft, Frankfurt/Main 1958; Carl Joachim Fnednch, Totalitäre Diktatur, Stuttgart 1957; zur Kntik und Reformulierung vgl. Martın Jänıcke, Totalitäre Herrschaft, Berlin 1971.

16 Fraenkel zituert eine Rede von Cari Schmitt vor dem Langnam-Verein (dem Verein der Rheinisch-Westfälischen Schwerındustrie) vom November I932: - Der qualitatıv totale Staat behalte alle entscheidenden Mittel der Massenbeeinflussung in der Hand. Neben diesem dem staatlichen Regal vorbehaltenen Bereich müsse es aber ım qualitauv totalen Staat eine Sphäre des freıen ındividuellen Unternehmertums und eine zwar nicht staatliche, aber öffentliche Sphäre geben « (S. 92). Entsprechend begrenzt war gegenüber diesem autonomen Bereich auch die *politische Gewalt< des Maßnahmestaates, was auch die NS-w Staatstheorie* immer wieder betonte (vgl. S. $128 \mathrm{ff}$.).

"Fraenkel S. 17I-18I; vgl. auch Ridder (FN 4), S. 236 f. Ridder wetst nach, daß dieser Versuch theoreusch schettern mußte, versäumt es aber im Gegensatz zu Fraenkel, den gesellschaftlichen Realitätsgehalt dieser Dokerin genauer zu bestimmen. 
schaftlicher und ökonomischer Autonomie ("Selbstverwaltung"), die entlang der alten Linien der Interessenorganisationen, mit der entscheidenden Ausnahme der Arbeiterklasse, errichtet wurden. Kein geringerer als Krupp hat den Trick des Ständestaates schnell durchschaut und eifrig an seinem Aufbau, mit allen Kompetenzen - wie es im Faschismus üblich war, mitgewirkt ${ }^{48}$. Die Beziehungen der Staatsgewalt zu diesen Bereichen gesellschaftlicher Autonomie und ökonomischer "Selbstverwaltung « bestand in einer Mischung aus Aufrechterhaltung der tradierten Formen des Zivil- und Verwaltungsrechts (eben des Normenstaates) einerseits (S. $128 \mathrm{f}, 218 \mathrm{f}$.) und zunehmenden direkten Urbereinkünften - man ist an den alten bürgerlichen, dem Gedanken der Aktiengesellschaft analogen Vertragstaat erinnert - bzw. direkter Unterwerfung zwischen den einzelnen Kapitalen (Zwangskartelle) und Kompromißbeziehungen zwischen diesen Komplexen und dem Staat andererseits (vor allem im Bereich der Rüstungswirtschaft) ${ }^{29}$.

Gegenüber dem Einzelnen in seiner abstrakten Qualität als Staatsbürger wurde ein differenziertes System der Integration und Beherrschung errichtet, welches vor allem die Arbeiterklasse erfaßte. Nach der Zerschlagung der Arbeiterorganisationen erhielt diese keme autonome Interessenvertretung mehr. Die Deutsche Arbeitsfront mit ihren sozialdemagogischen Funktionen ${ }^{j 0}$ bekam zwar mit dem Erreichen der Vollbeschäftigung nach $1936 / 37$ und den Problemen der Lohndifferenzierung etc. vorübergehend Kampfcharakter ${ }^{31}$, wurde aber immer wieder mit Macht aus jeder potentiell "gewerkschaftlichen" Rolle gedrängt. Als Arbeitskraft unterlag der Arbeiter nach der Abschaffung des kollektiven Arbeitsrechts und der Rückführung des individuellen Arbeitsrechts auf das bürgerliche Schuldrecht voll der Herrschaft des Kapitals. Als ehemaliger Staatsbürger wurde er nun als "Volksgenosse " wie alle anderen in das Netz der faschistischen Einheitspartei und ihrer Unterorganisationen und damit in die "Volksgemeinschaft " eingegliedert. Als potentieller Klassenkämpfer wurde er Objekt der staatlichen Verfolgung, ebenso wie andere Regimefeinde oder die Juden ${ }^{\prime 2}$. Der Nationalsozialismus begriff die Partei als »Trägerin des Volkswillens « und die Polizei als "Hüterin der Volksgemeinschaft " "3.

Die These von der Koexistenz von Maßnahmenstaat und Normenstaat, welche das faschistische Herrschaftssystem kennzeichne, hat Fraenkel an der Funktionsweise eines Teils der Staatsgewalt entwickelt, der Justiz. Die historisch-soziologischen Fundierungen dieser These führen dazu, das Auseinandertreten von zwei verschiedenen Herrschaftsmethoden als allgemeine Grundstruktur der politischen Systems unter dem Faschismus zu charakterisieren. Der Maßnahmenstaat als ein System der staatlichen Willkürherrschaft, charismatisch legitimiert durch und organisatorisch zentriert auf die "Führergewalt «, charakterisiert alle Institutionen im Faschismus, die bei der Feindbekämpfung eine Funktion hatten: Strafgerichte, Sondergerichte, Volksgerichtshof, Kriegsgerichte, Gestapo, SD und SS, und auch die Partei- und "Ständeinstanzen", die sich an der Denunziation und Verfolgung von Gegnern beteiligt haben. Die Definition des Normenstaates als eines Systems der Regelung

ss Vgl. Dieter Grosser, Die nationalsozualistzsche Wirtschaft, in: Das Argument, Nr. 32, 1965, S. 1-1 1, 7 ff., Schweitzer (FN 2), 239-296.

19 Vgl. Otto Kirchhemer, Die Rechtsordnung des Natzonalsozualismus (FN 1), S. $117,121 \mathrm{ff}$.

30 Ein schönes Besspiel völliger Ignoranz gibt Shirer (FN 2), der noch nachträglich an diese Demagogıe glaubt und in der Hoffnung auf den Volkswagen eines der stärksten Integrationsmittel des NS sieht (S. 258).

"V Vol. hierzu T. W. Mason, Labour in the Third Reach, in: Past \& Present, Nr. 33, 1966, S. I I 2-I I I und die Andeutungen in Der Prtmat der Politik - Politik und Wirtschaft tm Nattonalsozialismus, in: Das Argument, Nr. 41, 1966, S. 473-494, 485 f.

3 Zur Unierdrückung der Arbeiterklasse vgl. auch Neumann (FN I), S. 326-374 ( - The Ruled Classes«); Schweitzer (FN 2), S. 350-409.

"Buchheim (FN ro), S. 117. 
der Beziehungen zwischen »ökonomischen« Subjekten, Staatsbürgern (»Volksgenossen «), Parteimitgliedern u. a. und ihren jeweiligen staatlichen oder "halbstaatlich « organisierten Kontrahenten nach technisch rationalen, vorhersehbaren, nicht rückwirkend zu ändernden Regeln, trifft nicht nur auf die Justiz und deren Verfahrensweisen, sondern auch auf die staatliche Bürokratie, die Partei- und Selbstverwaltungsorganisationen zu.

Die Trennlinien zwischen diesen Methoden und ihren jeweiligen Trägern sind dabei fließend, gerade weil die »Führergewalt ", aus dem Belagerungszustand entstanden, sich verfassungsrechtlich nicht eindeutig festschreiben ließ. Während der parlamentarische Verfassungsstaat durch seine rechtliche Ordnung der Sphäre der Offentlichkeir (der Politik) durch Grundrechte, Organkompetenzen, Herrschaft des Gesetzes, Verantwortichkeit der Regierung etc. die Interesseneinflüsse prinzipiell von der geregelten Entstehung und Geltung verbindlicher staatlicher Normen trennt, war die Trennung des »Politischen " vom "Unpolitischen« - anders ausgedrückt die Trennung von Staat(sgewalt) und Gesellschaft - im Faschismus nicht mehr nach allgemeinen Regeln durchführbar. Gleichwohl mußte der Faschismus Maßnahmenstaat und Normenstaat miteinander verzabnen, wollte er nicht das Prinzip der Einheit der Staatsgewalt ganz aufgeben und zulassen, daß die für eine kapitalistische Gesellschaft notwendige Kalkulierbarkeit der staatlichen Aktionen völlig in die Brüche ging. Hierfür gab es zwei Praktiken. E. R. Huber hat eine beschrieben, die Legalisierung.

"Die Legalität bedeutet eine äußere Uberbrückung der Kluft, die in Wahrheit zweı wesensverschiedene Ordnungen trennt. Rücksicht auf das technısche Funktionieren des Justız- und Verwaltungsapparates sınd die eıgentlichen Gründe für die Methode der Legalität $\alpha^{\mu *}$.

Die andere Praktik bestand in der halb- und nichtöffentlichen ad-hoc-Ubereinkunft zwischen verschiedenen Machtträgern, die aus dem Mangel allgemeiner Abgrenzungen im Herrschaftssystem immer mehr notwendig wurden. Legalisierung zum Zwecke des Funktionierens staatlicher Verwaltung und ad-hoc-Ubereinkünfte zwischen verschiedenen Machtträgern, d. h. Interessengruppen, traten im Faschismus an die Stelle der Funktionen der öffentlichen Interessenartikulation und parlamentarischer Gesetzgebung im demokratischen Verfassungsstaat. Dieses ganze System wurde überwölbt durch die legitimitätsspendende Macht des »Führers«, dem so gewissermaßen die Funktion der Gesamtrepräsentation zukam"s. Die gesellschaftliche Funktion dieses Herrschaftssystems kann aber nur zureichend begriffen werden, wenn man es als eine Form bürgerlicher Herrschaft interpretiert, die sich zur "Totalität « nicht aufschwingen kann, in der die Grundstruktur der kapitalistischen Gesellschaft die Grenze jeglicher »außernormativer « Führergewalt ist.

III. Der Doppelstaat als Moment des Verbältnisses von Okonomie und Politik ım Faschismus

Mit dem Nachweis der Notwendigkeit der Koexistenz von Maßnahmen- und Normenstaat werden zwei Einwände entkräftet, die gegen Fraenkels These ins Feld geführt werden und die versuchen, von einer allgemeinen Theorie des NS-Herr-

14 Zit. nach Buchhem, S. 23. Vgl, auch F., S. 178; Buchheim interpretiert diese Legalisierung nur unter dem Aspekt nachträglicher scheinlegaler Begründungen der waußernormatıven Führergewalt . Er übersıeht, daß dies aucht nur ein Herrschafts-(Legitımations)tnck der Nationalsozialisten war, sondern die gesellschaftlichen Grundlagen des Doppelstaates betraf.

is Ridder ( $F N_{4}^{4}$ ), S. 230 f.; der sich daber vor allem auf Neumann und Kirchheimer bezieht. In diesen Zusammenhang gehört auch die NS-Gemenschaftsideologie, vgl. Fraenkel, S. 164-18 I, 221 f. 
schaftssystems aus seine Analyse ausschließlich auf einen Teil des Staates und ausschließlich auf einen bestimmten Zeitraum zu begrenzen.

Der erste Einwand ist von Buchheim prononciert vorgetragen worden. Für ihn erhebr sich die Frage,

nob die pure Maßnahme, das heißt: ob das Prinzip der außernormativen Führergewalt nicht dem Prinzip staatlichen Lebens derart entgegengesetzt ist, daß es nicht mehr unter dem Begriff staatı gefaßt werden kann. Im Selbstverständnis der Nationalsozialisten jedenfalls war das Prinzip der Führergewait etwas völlig anderes als Staatsgewalt, und in ihrer Herrschaftspraxis vollzog sich . . . ein fortschreitender Prozeß der ,Entstaatlichung des öffentlichen Lebens* ${ }^{36}$.

Hier wird die Bedeutung der Unterscheidung von demokratischem Verfassungsstaat und Klassenstaat sichtbar. Buchheim geht von zwei zentralen Mißverständnissen der Totalitarismustheorie aus. Zum einen wurde lange Zeit das Selbstverständnis des Nationalsozialismus als die Sache selbst genommen, das Prinzip des Führerstaates war dann schon auch dessen Realität. Zum zweiten fand die Aufarbeitung des Faschismus von einer prinzipiellen theoretischen Trennung von Politik und Okonomie aus und von der vorgefaßten Vorstellung her statt, daß der Faschismus mit der Abschaffung der parlamentarischen Demokratie auch mit der bürgerlichen Gesellschaft gebrochen habes ${ }^{37}$. Im Nachhinein konnte dann das Prinzip des $\bowtie$ Doppelstaates* zur bewußten oder unbewußten Exkulpation der bürgerlichen Gesellschaft verwandt werden. Aber auch dort, wo dies nicht die Absicht ist, wird gelegentlich in der Auflösung der Verfassung auch eine "Auflösung des 'Staates « «s gesehen.

Sofern man sich auf die Analyse der Herrschaftsorganisatzon alleine bezieht, kann man natürlich feststellen, daß der Nationalsozialismus die gesamte öffentliche Sphäre "total «durchorganisiert hat und im Rahmen der Feindbekämpfung sich jeder rechtsstaatlichen Nomierung entzog. Somit scheint der „Führerstaat $\propto$ alles andere zu dominieren. Wenn man die NS-Herrschaftsmethoden von den Matenen der Beherrschung trennt (und Buchheim sieht nicht, daß Fraenkel das Prinzip des Doppelstaates ja gerade von den Funktionserfordernissen der kapitalistischen Gesellschaft her entwickelt: "Nur wenn der wirtschaftliche Hintergrund des Regimes ausreichend berücksichtigt wird, ist verständlich, warum der Staat des heutigen Deutschland >doppelten Charakter besitzt", S. 203), wird das "Politische" im nachhinein ebenso objektlos wie es der faschistischen Theorie nach sein sollte. Nun wird aber neuerdings auch in der bürgerlichen Nationalsozialismusforschung immer mehr erkannt, daß der Faschismus die hergebrachten Institutionen und Formen der bürgerlichen Gesellschaft dann nucht abschaffte, wenn sie entweder für den Fortbestand lebenswichtig waren oder die von der Feindbekämpfung her, d. h. von der Unterdrückung der Arbeiterklasse, gebotenen "politischen« Maßnahmen nicht beeinträchtigten; daß andererseits die neuen Herrschaftsinstrumente die etablierten politischen Institurionen ntcht ersetzen konnten (die Partei nicht die Staatsbürokratie, die SA nicht die Reichswehr, die SS nicht einmal die Kriminal- und Ordnungspolizei, die SS-Wirtschaftsunternehmen nicht die kapitalistische Wirtschaft), son-

\footnotetext{
${ }^{6}$ Buchherm (FN ro), S. 21

$37 \mathrm{Zu}$ dieser Interpretation har zuletzt die Noltesche Faschismus-Theone beigetragen, der den Faschismus aus einer $*$ Krise des liberalen Systems* herleiret und damit das antibürgerliche Selbstverständnis des Faschismus für die Sache selbst nimmt. Vgl. ders., Die Krose des liberalen Systems und die faschistischen Bewegungen, München 1968; Der Faschismus in seiner Epoche, München 196s. Zur Nolte-Kritik vgl. Johannes Agnoli, Zur Faschismus-Diskussion I und II, in: Berliner Zestschrift für Politologıe (9) 1968, Nr. 2 und 4; Bernhard Blanke, Thesen zur Faschismus-Diskussion, in: Sozialistische Politik (1) 1969, Nr. 3; Richard Saage, Bemerkungen zur Faschusmustnterpretation Ernst Noltes, in: Das Argument, Nr. 58,1970 , S. 292-304; Reinhard Kühnl, Probleme einer Theone über den internationalen Faschusmus, Teil I: Die Faschismuskonzepuon Ernst Nolses, ın: Politısche Vierteljahresschrift (1 I) 1970, Heft 2/3, S. $318 \mathrm{ff}$.

s Ridder (FN 4), S. 234. Ahnlich Neumann (FN r), S. 383 f.
} 
dern neben ihnen existierten"s. Dieses Nebeneinander führt zu "pluralistischen "

Vorstellungen des NS, die die Totalitarismustheorie ersetzen, aber nach wie vor an der Trennung von Politik und Okonomie und an den Organisationsformen der Herrschaft orientiert sind.

Vor allem Franz Neumann (und zum Teil auch Fraenkel, vgl. S. 204 f., 216 f., 238 f.) konstatierte schon früh einen vertikalen Pluralismus von vier Säulen des faschistischen Herrschaftsystems (Partei, Staat = Bürokratie, Wirtschaft, Wehrmach $t^{+\infty}$ ), die nebeneinander und in kompromißhafter Abstimmung ihrer Interessen das Dritte Reich regierten. Neumann verbindet theoretisch die Analyse der Entwicklung des Kapitalismus zum Monopolkapital (Hilferding, Lenin u. a.) mit der Bürokratietheorie Max Webers". Seine Theorie bleibt auf die organisatorischen Trennlinien im Herrschaftssystem des Dritten Reiches und auf die strukturelle Analogie der Vermachtungsprozesse (Monopol, Bürokratie) beschränkt. Sie verfehlt wie alle Theorien, die von einer Art arbeitsteiliger Herrschaft ausgehen ${ }^{* 2}$, die funkttonelle Problematik und die funktionellen Unterschiede. Die gleichen Organisationen und Institutionen haben im Nationalsozialismus jeweils unterschiedliche Funktionen erfülit, die sich nicht nach Säulen aufteilen lassen. Die schier undurchschaubaren Kompromisse, Konflikte und Abmachungen, denen Umorganisationen folgten, waren Ausdruck dieses Problems. So hat der Terrorapparat der SS in seiner Eigenschaft als Arbeitskraftvermittler für die Rüstungsindustrie Funktionen der "Wirtschaft « ausgeübt, wäre also nach der Säulentheorie dieser zuzuordnen, so gerne die Herren aus den Direktionsetagen sich die Hände sauber halten wollten. Die „Partei« war auf dem Feld der Sozialintegration (DAF und andere Unterorganisationen) funktionell etwas anderes als im Bereich des Staatsapparates: der "Führer und Reichskanzler" war als Regierung ganz und gar Staat, selbst wenn sich die alte Ministerialbürokratie darüber mokieren mochte.

An der Justizentwicklung läßt sich dieses Problem noch einmal kurz skizzieren ${ }^{+3}$. In der Weimarer Republik sah sich eine "klassenbewußte" Justiz vor dem Problem, gewissermaßen in eigener Regie einen "Doppelstaat« zu errichten. Gegen den parlamentarischen Gesetzgeber und gegen die politische Linke praktizierte sie mittels richterlichem Nachprüfungsrecht und Ausnahmejustiz Durchbrechungen geltenden Rechtes und Einseitigkeiten der Rechtsprechung, die als "Klassenjustiz* zu einer Krise der - doch ihrem Anspruch nach - unabhängigen Justiz führte. Der Faschismus löste dieses Problem durch eine Beseitigung des Rechtsprechungsmonopols (den Maßnahmenstaat) und durch die Neuschaffung explizit politischer Gerichte wie etwa den Volksgerichtshof. Dadurch wurden die Gerichte »entlastet", ein großer Teil konnte sunpolitisch « die Bewahrung der kapitalistischen Rechtsgrundsätze garantieren (im Strafrecht gab es allerdings Uberschneidungen der Funktionen), während die "politische" Justiz als Sonderjustiz tätig war. Gleichwohl haben die ordentlichen Gerichte an dieser „Entlastung " tatkräftig mitgewirkt, solange die

19 Zuletzt Dand Schoenbaum, Die braune Revolution, Köln-Berlin 1968. Dies macht auch die nachträgliche Analyse der Terrororganisationen so schwer, wie die Arberten in Buchheim u. a. zur Anatomie des SS-Staates deutlich machen.

40 Neumann (FN 1); auch Reınhard Kühnl, Formen bürgerlicher Herrschaft. Liberalismus - Faschismus, Reinbek 197:, S. 1 30-ז 46 .

"Von eınem ähnlichen Ansatz geht auch Schweıtzer (FN 2), S. 9- 59 , aus. Zur Krıtik an solchen Ansätzen vgl. Eike Hennig, Thesen zur deutschen Sozual-und Wirtschaftsgeschrchte, Frankfurt/Main 1973, S. $30 \mathrm{ff}$.

«Vgl. Hennig (FN 4 I ), S. I25-136. Die Schwierigkeiten, den Klassencharakter des Faschismus empirisch im einzelnen nachzuweısen - wie Hennıg nchtıg anmerkt - darf nicht dazu führen, diesen Beweis nur auf der Ebene der Macht- und Interessenkoalition zu suchen. Dabet werden "Staat* und .Okonomie in ihrer jeweiligen formell-instrtutionellen Besonderung gegenemander einfach vorausgesetzt und ihr Verhältrus nıcht funktıonell bestımmt.

4) Vgl. in übrgen Fraenkel (FN 6), Brünneck (FN 4), Kirchheimer (FN 1), Perels (FN 22). 
Ausdifferenzierung des "Politischen* noch nicht hinreichend geklärt war, vor allem bei der Judenverfolgung. Auf anderen Gebieten (Kartellgerichte, Ehrengerichtsbarkeit) wurden gesellschaftliche Interessenkonflikte zwar noch formell juristisch, faktisch aber nach Machtgesichtspunkten oder opportunistischen Legitimitätsvorstellungen gelöst. Am deutlichsten war die Entlastung der Justiz im Bereich des Arbeitsrechts: da dieses fast völlig abgeschafft wurde, kamen die entscheidenden gesellschaftlichen Konflikte schon gar nicht mehr vors Gericht. Die Sorge, die Carl Schmitt in der Weimarer Republik hatte, daß die "politische Substanz a der falschen Stelle ist, wenn die Justiz politisch wird und damit ihre - auf der Unabhängigkeit beruhende - Legitimationskraft schwindet" ${ }^{\mu}$, wurde im Faschismus unnötig.

Neben der "Kompromißstruktur des NS-Herrschaftssystems, in der die Schwierigkeiten der Funktionsdifferenzierung zum Ausdruck kamen, macht noch ein anderes Fakrum die Säulentheorie problemarisch. Das NS-Herrschaftssystem war in ständiger »Bewegung*, was nicht auf die faschistische Ideologie zurückzuführen ist (und etwa in der Theorie von der "Permanenten Revolution" von Sigmund Neumann"su einer besonderen Variante der Totalitarismustheorie geführt hat), sondern auf die in der kapitalistischen Gesellschaft notwendige Form der Durchsetzung ökonomischer und gesellschaftlicher Veränderungen: die Konkurrenz. Die Konkurrenz wurde vor allem von Mason* als eine wesentliche Kategorie auch des politischen Herrschaftssystems im Nationalsozialismus hervorgehoben. Dem Verständnis dieses Momentes steht allzu oft ein Begriff von "Monopolkapital (sowohl bei Neumann schon als auch in der DDR-Theorie) gegenüber, das Konkurrenz als "freie* und Monopolkapital entgegensetzt. Ungeachtet der tatsächlichen Veränderungen des Kapitalismus durch Konzentration und Zentralisation muß festgehalten werden, daß die Art und Weise, in der sich im Kapitalismus die Verwertung des Kapitals und damit Strukturveränderungen der gesellschaftlichen Reproduktion durchsetzen, die Konkurrenz bleibr, wie groß auch die Einzelkapitale immer sein mögen, wie mächtig sie im einzeinen durch Konzentration werden. Was sich ändert sind die Formen der Konkurrenz.

$\mathrm{Da}$ der Faschismus alles andere als eine Planwirtschaft war, auf der anderen Seite aber in vielen Gebieten reglementierend eingriff, nahm die Durchsetzung und Artikulation von Interessengegensätzen in diesen Bereichen die Form der Konkurrenz innerhalb und zwischen den "Selbstverwaltungsorganisationen ", deren Pendants in der Partei- und Staatsverwaltung, in der DAF oder der SS etc. an. Hatte die Abschaffung des Parlamentarismus die Interessenkonkurrenz zwar von der Uberlagerung durch Interessen der Arbeiterklasse sentmischt « und schien daher die Beziehung von Kapital und Staat direkt und ungetrübt, so brachte die faschistische Partei mit ihrer Funktion, das Gesamtsystem plebiszitär (und auch das ist eine Form von Offentlichkeit, wie pervertiert aus demokratischer Sicht auch immer) zu legitimieren, die entpolitisierten und unterdrückten Interessen verzerrt wieder ins Kompromißsystem ein: sei es in dem Versuch, den Lebensstandard im "Altreich" bis in den Krieg hinein noch auf dem - ohnehin niedrigen - Vorkriegsniveau zu halten; sei es in der terroristisch-repressiven Seite der Integration, der »Rasseideologie «. Die Schachtschen Versuche (S. I I $\mathrm{ff}$.), den Antisemitismus aus dem autonomen Bereich der Wirtschaft herauszuhalten, mußten mit der forcierten Kriegsvorbereitung und der damit einhergehenden Hysterisierung der inneren Feindbekämpfung scheitern.

4 Carl Schmite, Der Hüter der Verfassung, Berlin 1931, bes. S. 48-70.

4S Sigmund Neumann, Permanent Revolution, London ${ }^{2} 1965$.

6 Mason (FN 31 ), S. ${ }_{4} 82$ ff. 
Die Hauptprobleme der Konkurrenz im Bereich des Staates ergaben sich aus der Abschaffung der "freien Märkte" als Form der Konkurrenz für Geld und Kredit, Rohstoffe und Außenhandel, und auch für die Arbeitskraft. Die hier eingeführten "staatlichen « Reglementierungen (Neumann hat für diesen Bereich den Begriff der »Befehlswırtschaft « geprägt") waren in den meisten Fällen Resultat einer Zusammenarbeit von Kapital und Staatsbürokratie oder Wehrmacht. Damit wurde aber die Konkurrenz eine Konkurrenz zwischen den politischen Institutionen selbss ${ }^{8}$. Deshalb scheint es nahezuliegen, diese - aus der Unklarheit der Trennlinien zwischen Staat und Gesellschaft, Politik und Okonomie erwachsenden - Konkurrenz- oder Entdifferenzierungsprozesse seien entweder ein raffinierter Herrschaftstrick der Nazis (so in der Totalitarismustheorie) gewesen oder hätten zwangsläufig zu einer „Entscheidungsüberlastung " der Herrschaftszentrale geführt". Mason hat dies zur These von "Primat der Politik « ausformuliert".

Hieraus resultiert der zweite mögliche Einwand gegen Fraenkel: sein Bild von der Koexistenz von Maßnahmenstaat und Normenstaat gebe nur die Struktur des Herrschaftssystems in der Anfangsphase bis etwa 1938 wieder ${ }^{5-2}$. An dieser Sicht sind zwei Momente richtig: der nationalsozialistische Staat hat (wobei immer analysiert werden muß, welche Funktion noch in den tradierten Formen der ökonomischen Autonomie und welche mittels bürokratischer Úbereinkunft und staatlicher Sanktionierung gelöst wurde) nach 1936 die Dimension seiner direkten Reglementierungen ökonomischer Prozesse ebenso erweitert wie sich die Institutionen des politischen Maßnahmenstaates ausgedehnt haben. Kann man deshalb von einem "Machtzuwachs" der Partei sprechen, wie es eine an der Säulentheorie orientierte Faschismustheorie tut's ? Systemtheoretisch gesprochen kann man gegen diese These einwenden, daß bei der Uberlastung der zentralen Entscheidungsinstanz, die zu krisenhaften Nichtentscheidungen oder dysfunktionalen Entscheidungsprozessen führt, sich zur Lösung der Probleme zwangsläufig "funktionale“ Aquivalente «" ausbilden, im Faschismus in Gestalt zahlreicher »Unterregierungen « (der Ausdruck wurde im heutigen Italien - sotto gioverno - geprägt, um die Tatsache der Verfilzung des Kapitals mit der DC und dem Staatsapparat unterhalb der parlamentarischen Kontrolle zu bezeichnen), die quer und gegen die offiziellen Entscheidungsstrukturen funktionierten. Gerade solche Querverbindungen haben sich in der Zeit zwischen 1937 (Amt für den Vierjahresplan) und 1942 ausgedehnt. Die Struktur der Koexistenz von Maßnahmenstaat und Normenstaat hat sich sicher verändert, als die beiden wesentlichen Methoden blieben sie jedoch konstitutiv für das nationalsozialistische Herrschaftssystem.

Mit der forcierten Knegsvorbereitung muß in den Begriff des Maßnahmenstaates

4 F. Neumann (FN I), S. 240-296.

4. Vgl. Blanke (FN 37).

44 Konrad Schacht/Lutz Unterseher, Versuch zur Systematsszerung des Chaos, in: KJ 1970, S. 9-2I.

s Mason (und in semer Nachfolge auch neuere Varıanten der Totalitarismustheone, vgl. etwa Martin Broszat, Der Staat Hitlers. Grundlegung und Entwicklung seiner inneren Verfassung, München 1969) entwickelt diese These auf der Grundlage der außenpolitischen, auf den Krieg zusteuernden Dynamik des Faschismus. Vgl. hierzu Teil IV dieser Rezension.

ios Vgl. Bracher/Sauer/Schulz, Die nationalsozvalistische Machtergreifung, Köln-Opladen 1963, S. 175, $373 \mathrm{f}$.

"So vor allem Schweizzer (FN 2, S. $504-583$ ), der den Faschismus in die beiden Phasen des "partial fascism $\propto$ und des ofull fascism* unterteilt. Vgl. meine Rezension in: Das Argument, Nr. 32, 1969, S. $42-44$.

1. Der Begriff ist von Robert K. Merton in den sozıologıschen Funktıonalismus eingeführt worden und bezeıchnet die Möglichkeıt, bestımmte Funktıonen durch verschıedene Prozesse und Strukturen ersatzweise zu erfüllen. Einıge Überlegungen zur Ausbildung funktıonaler ̊̊quvalente um Faschısmus finden sich bel SchachvUnterseher (FN 49); vgl, auch Bernhard Blanke / Ulrich Jürgens / Hans Kastendiek, Zur neueren marxistiscben Diskussion über die Analyse von Form und Funktion des bürgerlichen Staates, In: Probleme des KJassenkampfs, Nr. 14/15, 1974, S. 51-102, 100. 
allerdings die Rüstungsvorbereitung (etwa auf dem Gebiet der Arbeitskräftelenkung) einbegriffen werden. Die äußere Feindbekämpfung trat an die Seite der inneren und veränderte die Methoden der Zusammenarbeit zwischen Staat und Wirtschaft, wie sie den Ausbau des traditionellen Maßnahmenstaates der inneren Feindbekämpfung forcierte. Aber mit dieser Ausdehnung entstand nicht endlich der "totale $\propto$ Staat, sondern zunächst nur ein organisatorisches Chaos. Soweit dieses aus den Herrschaftsansprüchen des Nationalsozialismus resultierte, wirkte es sich im Bereich der »Politik * aus, d. h. in der Staatsverwaltung den Instanzen der Interessenvermittlung und -integration und der Legitimation (Partei und ihre Unterorganisationen) und teilweise der Feindbekämpfung, soweit diese die weitere Herauslösung etwa der SS und der Sondergerichtsbarkeit aus dem traditionellen Gewaltapparat betraf. Soweit das Chaos die Umorganisationen in der Okonomie betraf, hatte es andere Ursachen und Folgen als den endlich durchgesetzen Machtanspruch der faschistischen Partei. Die Probleme der ökonomischen Reproduktion resultierten aus den Zwängen der Kriegsvorbereitung, die selbst wiederum ein tiefergehendes Problem reflektierten, in dem überhaupt die ganze Ursache des Faschismus lag, nämlich den im Grunde erst Anfang der soer Jahre überwundenen, in der Weltwirtschaftskrise zum Ausdruck kommenden Umgruppierungsvorgängen im System des Kapitalismus im Weltmaßstab.

Die Frage nach dem Zusammenhang zwischen Kapitalismus und Faschismus entscheidet sich somit an der Frage, ob der Krieg das Produkt der Nazi-Strategie war, in die das Kapital nur widerwillig und allein der höheren Profite wegen eingestimmt hatte, oder ob der Zweite Weltkrieg selbst Produkt des Imperialismus war. In der bürgerlichen Faschismustheorie wird immer noch versucht, den Kapitalismus vom Faschismus dadurch reinzuwaschen, daß entweder das Fehlen von direkren Einflüssen schon bei der Machtergreifung nachgewiesen wird"s oder dies wenigstens für die unmittelbare Kriegsstrategie behauptet wird. In der DDR-Theorie versteifte man sich jahrzehntelang auf die Widerlegung dieser Behauptungen durch den aktenkundigen Nachweis solcher Beziehungen, sei es bei der Machtergreifung, sei es bei der Kriegsvorbereitung, etwa beim Amt für den Vierjahresplan ${ }^{\text {s4 }}$. So wichtig dieser Aspekt auch sein mag, er verfehlt letztlich die Frage nach dem Zusammenhang des Kapitalismus als System mit der spezifischen faschistischen Herrschaftsform.

\section{Gesellschaftliche Krise und politischer Ausnahmezustand}

Die Frage nach dem Systemzusammenhang wird bei Ernst Fraenkel ansatzweise beantwortet. Wenn er den Faschismus als den Staat des permanenten Belagerungszustandes bezeichnet, macht er diesen zwar an den Veränderungen der Verfassungsinstitutionen fest, aber in der Logik der Feindbekämpfung entdeckr er doch die Ursache für die institutionellen Veränderungen, die größte Krise des kapitalistischen Systems, und zwar im inneren wie auf dem Weltmarkt. Alle Faschismuserklärungen müssen von dieser Krise ausgehen". Dabei ist aber wichtig, daß die Krise nicht in den Etappen der politischen Geschichte analysiert wird: daß nicht etwa der Januar 1933 als wesentlicher *Wendepunkt« genommen wird, dann das Jahr 1939 und

"So neuerdings von Henry Ashby Turner jr., Faschismus und Kaputalismus in Deutschland, Görtungen 1972 (das besser heißen müßte: der Faschismus und die Kapitalisten); Gerhard Schulz, Aufstieg des Nationalsozialismus, Berlin 1975; zu anderen Nachwersen vgl. Hennig (FN 4I), S. 34 ff.

" Vgl. die Kontroverse in: Das Argument, Nr. 47, $1968 \mathrm{zw}$ ischen Mason, Czichon, Eichholtz und Gossweiler.

"So auch Kühnl (FN 40 ), S. 77 f., 99 f. 
schließlich der Zusammenbruch. Die Totalitarismustheorie orientierte sich bei der

Vorstellung von der "permanenten Mobilmachung" ausschließlich an den Daten des Formenwechsels im Bereich des politischen Systems und an den Haupt- und Staatsaktionen auf dem Gebier der Weltpolitik. Analog dazu orientierte sich die Darstellung des Nationalsozialismus als Herrschaftssystem an der Entwicklung des Terrors und der Terrorinstrumente. „Das Leben im Dritten Reich«, wie es Shirer sinnig nennt ${ }^{\text {s。}}$, verschwand völlig aus dem Blickfeld. So werden die Etappen der ökonomischen Entwicklung nach den Etappen der Planungen in der Rüstungswirtschaft analysiert: $1933 / 34$ bis $1936 / 37$ (Vierjahresplan), 1938 bis 1942 (Ministerium Speer/Stalingrad), 1942 bis zum Zusammenbruch. Markierungspunkte sind dann die Vollbeschäftigung, mit der die Krise als überwunden erachtet wird, Rüstungswirtschaft in auf die Blitzkriegsstrategie dimensionierter Form, totale Rüstungswirtschaft ab 1942 (auch Fraenkel geht ähnlich vor, vgl. S. 208 f.).

Die Probleme der kapitalistischen Reproduktion unter faschistischer politischer Herrschaft lassen sich jedoch zureichend nur vom Verständnis des deutschen Kapitalismus als Teil des Kapitalismus als Weltsystem ( Imperialismus $«$ ) her begreifen. Eine solche Theorie liegt noch nicht vor. Den bislang besten Ansatz hierzu hat m. E. Alfred Sohn-Rethels7 geliefert (im übrigen auch aus einer Binnenperspektive, er hatte bis 1937 als Hilfskraft in einer Informationszentrale des Kapitals Einblick in die Interessenkämpfe und Strategien des deutschen Kapitals). Seine Argumentation soll hier kurz aufgegriffen werden.

Der deutsche Kapitalismus - durch den verlorenen ersten Weltkrieg ohnehin in seinen Akkumulations- und Expansionsmöglichkeiten eingeschränkt - war durch die Weltwirtschaftskrise besonders stark getroffen. Um die Akkumulation wieder in Gang zu setzen, waren zwei Probleme zu lösen: einmal mußten die gerade von einer reformistischen Arbeiterbewegung (gewissermaßen als »Ersatz « für die nicht durchgeführte Revolution von I9I8/19) erkämpften Positionen rückgängig gemacht und die Bedingungen für eine rapide Erhöhung der Mehrwertrate hergestellt werden; zum zweiten mußten die aus der Weltwirtschaftskrise resultierenden divergierenden Interessen der Kapitale und der Großagrarier zu einem Ausgleich gebracht werden. Beide Aufgaben konnten die noch an den demokratischen Verfassungsstaat als allgemeinen Rahmen gebundenen Präsidialkabinette nicht lösen. Entscheidend ist aber, daß die Lösung bezder Probleme nur in einer Linie möglich war: die Wiederingangsetzung der Akkumulation durch terroristische Unterdrückung der Arbeiterklasse war sowohl Voraussetzung einer neuen Strategie wie sie zugleich an die veränderte Weltmarkt -, d. h. Expansionsstrategie des Kapitals gebunden war. Die Expansionsstrategie (in Richtung einer mittelosteuropäischen Großraumpolitik) wurde aber, je mehr der Weltmarkt zerfiel, eine Norwendigkeit auch für jene Teile des Kapitals, die aus der Krise noch relativ stark hervorgegangen waren. Die Lösung des Agrarproblems konnte unter diesen Bedingungen auf Kosten der unmittelbar für den Konsum produzierenden kleinen Landwirtschaft und zugunsten der militärstrategisch wichtigen, kapitalistisch produzierenden Großagrarier erfolgen. Die Reichswehr mußte von der "Rache für Versailles« nicht erst überzeugt werden.

so Shirer (FN 2) verwendet - und darn ist sein Buch prototypısch für alle Populärdarstellungen wıe Schulgeschichtsbücher - ganze I 8 von rund $\mathrm{x} 200$ Seiten auf dieses $*$ Leben $*$, ansonsten besteht für ihn der deutsche Faschismus aus politischer Machtergreifung, Terror und Außenpolicik.

"FN r. Von der Weltwirtschaftskrise geht auch die matenalreıche Arbeıt von Henng (FN 41 ) aus. Für die folgenden Thesen stütze ich mich auf die bereits zitıerte Literatur, die im einzelnen nicht mehr erwähnt wird. Daneben müssen noch herangezogen werden: Rene Erbe, Die nationalsozualistusche Wirtschaftspolitik 1933-1939 im Lichte der modemen Theone, Zürıch I958; Kurt Gossweiler, Großbanken, Industremonopole, Staat. Okonomie und Politik des staatsmonopolistischen Kapitalismus in Deutschland I9I4 bis 1932, Berlin (DDR) I971; Dieter Petzına, Autarkiepolitik ım Drtten Reich, Stuttgart 1968. Vgl. .m übrigen die Literaturangaben beı Hennig. 
Unterhalb der machtpolitischen und institutionellen »Übereinkunft« der verschiedenen Fraktionen der herrschenden Klasse existierte also am Ende der Weimarer Republik eine materielle Identität ihrer "Interessen ", die aus der ökonomisch zwingend gewordenen Krisenlösungsstrategie des deurschen Kapitalismus resultierte. Da diese Strategie die Unterdrückung der Arbeiterklasse voraussetzte, war die terroristische Diktatur der Nationalsozialisten nur die "logische* Konsequenz, nachdem eine revolutionäre Lösung der Krise aufgrund der Zersplitterung und der Schwäche der deutschen Arbeiterbewegung nicht möglich gewesen war. Nun bedeuter diese materielle, $d$. h. funktionelle Identität der $»$ Interessen $\approx$ der verschiedenen Herrschaftsträger in Okonomie und Politik keineswegs eine lückenlose Ưbereinstimmung ihrer Handlungen im einzelnen oder über die jeweiligen Maßnahmen und Etappenbestimmungen im Rahmen einer perspektivisch gleichlaufenden Strategie. Wer diese sucht - oder verneint - hat das zentrale Problem immer schon verfehlt. Eine Systemanalyse der Reproduktionsprobleme des deutschen Kapitalismus nach der Weltwirtschafrskrise - ökonomischer wie politischer Art muß unterscheiden $\mathrm{zwischen}$ den aus der gesellschaftlichen Totalität resultierenden allgemeinen $Z$ wängen in einer bestimmten historischen Phase und der Art und Weise wie sich diese $Z$ wänge auf der Ebene der "gesondercen $\kappa^{38}$ gesellschaftlichen Bereiche oder politischen Institutionen und in deren wechselseitigem Zusammenspiel jeweils durchsetzen.

Wenn sich deshalb im Verlaufe der Entwicklung nach 1933 immer wieder Krisen* einstellten, die eine Umgruppierung der Machtgruppen oder der institutionellen Organisierung der Krisenlösung und der Rüstungswirtschaft erzwangen, so sind daraus nicht nachträgliche - hypothetische - Alternativen zu konstruieren, ob das Kapital nicht vielleicht doch die Faschisten noch hätte loswerden können oder umgekehr die Faschisten nicht vielleicht doch die Herrschaft über das Kapital gewonnen haben. Der imperialistische Krieg war gewissermaßen in den Strukturdaten und den Entwicklungslinien von $1929 \mathrm{ff}$. vorprogrammiert - und hätte nur durch eine sozialistische Revolution in Deutschland verhindert werden können'? Einmal in Gang gesetzt, war die Entwicklung irreversibel geworden, die allgemeine Tendenz der gesellschaftlichen Entwicklung bestimmte nun das »Verhalten « der Akteure im Bereich der ökonomischen, sozialen und politischen Bewegung. Somit war die faschistische "Führergewalt « im doppelten Sinne Produkt einer Krise: sie war notwendig, um den Umbau des politischen Systems zum Zwecke der terroristischen Unterdrückung der Arbeiterklasse und der allgemeinen Militarisierung der Gesellschaft durchzuführen; sie war zugleich Produkt eines Zerfalls des Weltmarktes und mit ihm des Zerfalls der nach 1918 gegründeten internationalen Systembeziehungen, auch in diesem Felde war sie »Ausnahmegewalt«, nämlich Kriegsgewalt (vgl. die Kritik Fraenkels an der NS-Völkerrechtstheorie, S. 97, г 37 f., 169). Und das Kapital konnte die Faschisten nicht los werden, wenn es nicht gelang, die wirtschaftliche Entwicklung im Rahmen eines relativ freien Weltmarktes zu "normalisieren $\times$. Die Nationalsozialisten konnten gar keine andere Politik machen als die der Weltkriegsvorbereitung mit allen inneren wie äußeren Konsequenzen. Daran mußten auch alle "Normalisierungsversuche" scheitern, die während der NS-Herrschaft immer wieder stattfanden und heute gern als Zeichen des innerbürgerlichen Widerstandes gewertet werden. So werden die Jahre $1936 / 37$ gerne als "Entscheidungsjahre« dargestellt. Die Vollbeschäftigung war erreicht, was hätte

18 Vgl. hierzu Blanke/Jürgens/Kastendiek (FN 52); Joachim Hirsch, Staatsapparat und Reproduktion des Kaputals, Frankfurt/Main 1974, Teil I.

s9 Insofern hat Opıtz (FN 10) mit seiner Kntik an früheren Thesen von mir (FN 37) reche, er selbst löst das Problem mit dem Verweis auf die $*$ Verantwortung des Monopolkapitals* (S. 572) aber keineswegs. 
näher gelegen als - wie es aus Kreisen der Konsumindustrie damals gefordert wurde - auf »Friedenswirtschaft « umzuschalten und sich dem Weltmarkt wieder zu öffnen? Doch zu diesem Zeitpunkt erlebte die Weltwirtschaft gerade einen zweiten Einbruch, der auch das New Deal-Experiment in den USA traf. Eine andere Strategie hätte das deutsche Kapital erneut vor die Probleme gestellt, die es mit der Uberwindung der Folgen der Weltwirtschaftskrise aufgrund nationalstaatlicher Abkapselung gerade überwunden glaubre: die erneute Krise und die Unmöglichkeit, die Arbeiterklasse wieder mittels integrationistischer Tarif- und Sozialpolitik zu befrieden. Ein relevanter innerbürgerlicher Widerstand gegen den Faschismus war unter der Drohung der letzten Weimarer Frontstellungen unmöglich geworden.

Die an der Theorie vom Monopolkapital orientierte Faschismustheorie verweist an diesem Punkt gerne auf eine Machtverlagerung innerhalb der Fraktionen des Kapitals die zur Fortsetzung der Kriegspolitik geführt habe, vor allem auf die Dominanz der IG-Farben-Industrie unter dem Vierjahresplan. So richtig dieser Hinweis für die Widerlegung der Theorie vom gesteigerten Machtzuwachs der Nationalsozialisten ist, so unzureichend ist er, um die allgemeinen Tendenzen der Expansionsdynamik des NS zu erklären. Aber auch die besonderen Probleme, die sich nach 1936/37 stellten, werden mit dieser auf institutionelle Machtbeziehungen fixierten Theorie nicht erklärt. In der Tat stellt nämlich dieser Zeitpunkt einen "Wendepunkt « dar, wenn man in Betracht zieht, daß die kapitalistische Reproduktion nicht per se auf Rüstung hin strukturiert ist. Zwar konnten die Uberkapazitäten nach 1932 vor allem durch die Ingangsetzung der Rüstungsproduktion wieder voll genutzt werden, eine "militarisierte" Wirtschaft war in Deutschland damit noch keineswegs hergestellt. Unter den Bedingungen der Abschließung vom Weltmarkt und unter den Bedingungen der bis 1936 inganggesetzten spezifisch faschistischen Konjunktur mußte so zwar einerseits der eingeschlagene Weg fortgesetzt werden, dieser erzwang aber zugleich relevante Umgruppierungen. Die Rüstung hatte nämlich die Disproportionalität der kapitalistischen Reproduktion nur noch verstärkt. Die Finanzierung des Rüstungsprogramms hatte eine versteckte Inflation erzeugt, die im Vergleich der Währungen auf einem offenen Weltmarkt $t^{\infty}$ unmittelbar zu Tage getreten wäre. Die faschistische Konjunktur beruhte alles in allem auf der durch staatliche $\mathrm{K}_{r}$ editfinanzierung vorweggenommenen Ausplünderung künftig besetzter Gebiete. Dies sind die entscheidenden Momente, die auch die Veränderungen im Herrschaftssystem zur Folge hatten.

Wenn man Etappen in der Entwicklung des deutschen Faschismus abstecken will, dann sind sie somit vom Verlauf der Krisenlösung und deren jeweiligen Schwerpunkten her zu bestimmen. Auch die Entwicklungen im Herrschaftssystem des Nationalsozialismus sind dann als Ausdruck der latenten, durch die faschistische Organisation der Massen nur übertünchten, gesellschaftlichen Krise zu bestimmen. Eine der Logik gesellschaftlicher Krisenentwicklung und -lösung folgende Phaseneinteilung müßte foigende Einschnitte machen:

r. Die Ära Brüning, die den Anfang vom Ende des demokratischen Verfassungsstaates darstellt, auf dem Hintergrund der voll ausbrechenden Weltwirtschaftskrise ${ }^{6}$.

2. Die Zeit vom Sturz Brünings bis zum Herbst 1934, mit dem "Umbau " des politischen Systems, der Auflösung des Systems der reformistischen Integration der

- Vgl. Chnstel Neusüß / Bernhard Blanke / Elmar Altvater, Kapttalistischer Weltmarkt und Weltwährungsknse, in: Probleme des Klassenkampfs, Nr. I 1971; Chrisrel Neusüß, Imperalismus und Weltmarktbewegung des Kapitals, Erlangen 1972.

6. Insofern hat Arthur Rosenberg recht (Geschichte der Wermarer Republik, Frankfurt/Man I961), wenn er das Ende der Weimarer Republik mit 1930 ansetzt. 
Arbeiterklasse unter Papen und ihrer endgültigen Unterwerfung durch die Faschisten, dem Ende des faschistischen »Radikalismus (SA) und der »Verstaatlichung * des Nationalsozialismus, der erfolgreichen Reorganisation der kapitalistischen Wirtschaft (Zwangskartellierung, Schacht etc.), vor allem der staatlich organisierten Abschottung zum kapitalistischen Weltmarkt.

3. Die Zeit bis 1938, die schließlich die Durchsetzung der "Militarisierung* der Wirtschaft - auf der Grundlage des eingeschlagenen Wegs - bringt, mit ihren Umorganisierungen im Bereich der Rüstungswirtschaft (Vierjahresplan) und vor allem mit der Reorganisierung des Terrorapparates der SS und der Polizei, schließlich der Ausschaltung traditional orientierter Kreise in der Reichswehr.

4. Die Zeit von 1938 bis 1942 , d. h. die imperialistische Politik auf den Krieg zu bis zur "Wende" von Stalingrad, bestimmt durch ständige Umgruppierungen in der Organisation der Rüstungswirtschaft (was auch die noch an traditioneller Interessenpolitik orientierten "Selbstverwaltungsorgane * des Kapitals betraf), und des Herrschaftssystems insgesamt.

5. Die Zeit des totalen Kriegs, gekennzeichnet durch eine in der Regie des Kapitals selbst organisierte Rüstungswirtschaft (Ministerium Speer), begleitet durch den Ausbau des SS-Staates mit KZ's und Judenvernichtung. Zunächst entsteht ein "Doppelstaat « ganz eigener Prägung, definiert durch die Grenzlinien zwischen dem "Altreich « und den besetzten und Kriegsgebieten, wo sich der SS-Staat in seiner ganzen technisch-kalkulierten Grausamkeir ${ }^{62}$ entfaltet. Je mehr aber Deutschland selbst Kriegsgebiet wird, desto mehr schlägt der totale Krieg auch nach innen in jenen "totalitären« Staat um, der bis heute das apokalyptische Bild des deutschen Faschismus im allgemeinen Bewußtsein geprägt hat.

Erst in den beiden letzten Phasen, die Fraenkel nicht mehr analysiert hat, wird jene von der Ambivalenz einer faschistischen Herrschaft innerhalb einer bürgerlichen Gesellschaft geprägte - Koexistenz von Maßnahmenstaat und Normenstaat relativiert und der Normenstaat in seiner Bedeutung für die politische Reproduktion des Herrschaftssystems immer mehr verdrängt.

\section{Die $\star$ Einheitsfront der Naturrechtler $*$ und das Vorbild der $*$ westlichen Demokra- tzen}

Ernst Fraenkel hat diese Entwicklung prognostiziert. Konstatiert er für die ersten Phasen des NS noch die Koexistenz von Irrationalität (Maßnahmenstaat) und technischer Rationalität (Normenstaat), so weiß er doch, daß sich die innere Logik des Faschismus auf den Krieg zubewegt, daß die "ziellose Irrationalität« (S. 239 f.) des Faschismus nur das Ziel der totalen Destruktion haben kann. „Der Doppelstaat ist die notwendige politische Erscheinungsform einer an Spannungen reichen $\mathrm{Zwi}$ schenperiode», da der Kapitalismus bereit ist, sich »jeder substanziellen Irrationalität anzupassen, wenn nur die wichtigen Voraussetzungen für seine technisch-rationale Ordnung erhaiten bleiben* (S. 24I). Noch vor der Durchsetzung des SS-Staates, d. h. dem inneren "Ziel « dieser Zwischenperiode, spürt Fraenkel als Jude im Dritten Reich aufgrund der Bedrohung durch den »bürgerlichen « (S. 126) und den physischen Tod das kommende grausige Ende. Den Appeasement-Politikern aller couleur schreibt er ins Stammbuch:

64 Vgl. hierzu das ebenfalls noch nucht übersetzre Buch von Raul Hilberg, The Destruction of the European Jews, Chikago r96x, der die Gesamtheir der Vernuchtungsvorgänge analysiert und die notwendige Beteiligung sämtlicher Bürokratıen (Gerichte und Gesetzeskommentatoren) an eınem solchen •Unternehmen « feststellt. Vgl. auch das (neu aufgelegte) Buch von Eugen Kogon, Der SS-Staat, München 1946.

${ }^{6}$ Fraenkel (FN 7), S. 279 f., 295 f. 
"Zu Begınn seıner Herrschaft gab der Natıonalsozıalismus vor, aus staatspolitischen Gründen gegen den Kommunismus vorgehen und die kommunistische Parteı hors la lot stellen zu müssen. Nicht ahnend, wohin es führen werde, wenn ein partielles Rechtsvakuum geschaffen wird, gaben nur allzu viele hierzu ihre Zustimmung und bekannten sich mit wahrer Inbrunst zu dem Dogma ailer gleichgeschalteten Opportunisten, daß, wo gehobelt wird, auch Späne fallen* (S. 86).

Durch diese Erkenntnis und durch seine Kritik am Reformismus der Weimarer Sozialdemokratie und der Gewerkschaften (die die Tendenz hatten, "sich in Staatsorgane zu verwandeln «, S. 207) ist Fraenkel zwar nicht zum "Kommunisten " geworden, aber er teilt Rosa Luxemburgs Alternative "Sozialismus oder Barbareiк.

Wie ist dann seine Kehrtwendung nach 1945 zu erklären? Der New Deal, den er bewunderte, hat doch den amerikanischen Arbeitern nicht mehr gebracht als die reformistische Sozialdemokratie den deutschen in der Weimarer Zeit. Und der amerikanische Kapitalismus war vor faschistoiden Prozessen (wie etwa dem Mc Carthyanismus) ebenso wenig gefeit wie der deutsche. Aus Fraenkels Schriften während des Krieges lassen sich zwei Momente herauslesen, die ihn nach I945 fast auf den rechten Flügel der Sozialdemokratie geführt haben. In seinen Uberlegungen zum Neuaufbau der deutschen Gewerkschaften ${ }^{63}$ kommt die Enträuschung über die deursche Arbeiterklasse zu Tage, die er nach 2 Jahren Drittem Reich als zu großen Teilen von den Ideen des Nationalsozialismus infiziert bezeichnet und der er deshalb keine autonomen sozialistischen Aktionen zutraut. Zum andern ist es die Enttäuschung über die Entwicklung der UdSSR unter Stalin mit der Konsequenz des scharfen Antikommunismus.

Das sind jedoch nur die auf die Linke bezogenen politischen Faktoren. Hinzu kommt - und dieses Moment wird im "Doppelstaat $\propto$ besonders deutlich - eine wesentliche Uberlegung, die sich schon bei Hermann Heller vor 1933 findet: daß es in Anknüpfung an die klassischen bürgerlichen Ideen der Aufklärung, die er vor allem in den angelsächsischen Ländern verwirklicht sieht, gelingen müßte, eine "Einheitsfront der Naturrechtler* gegen die Barbarei zu bilden, die auch und vor allem noch das liberale Bürgertum umfaßt. Hier diente später die demokratische Koalition des New Deal als Vorbild. Die Erörterungen im "Doppelstaat« zum Problem des Naturrechts (S. 136-1 8r) kreisen um diese Frage. Als Leitmotiv kommt hier für die Arbeiten Fraenkels nach 1945 schon eine Idealisierung der sogenannten "westlichen Demokratien« zum Vorschein. In dem späteren Sammelband "Deutschland und die westlichen Demokratien $\star^{64}$ ist Fraenkel dann der Linie nachgegangen, die seine Naturrechtsüberlegungen im "Doppelstaat» bestimmte. Die deutsche Entwicklung erscheint nur noch als eine Sonderentwicklung, die es versäumt habe, den großen westlichen Demokratien nachzueifern und die deshalb nach dem Faschismus nur noch ein Ziel haben könne, nämlich sich endlich diesem Weg anzuschließen.

Im "Doppelstaat* (und in Arbeiten zum Begriff des Rechtsstaats während des Krieges ${ }^{6 s}$ ) wird diese Entwicklung an zwei markanten Punkten vorbereitet. Bei der Erörterung der Bedeutung des Ausnahmezustandes vergleicht Fraenkel die englische und amerikanische Regelung mit der deutschen Tradition. Er verweist vor allem darauf, daß dort die „Prärogative«, d. h. die unumschränkte Souveränität des Staates (ehemals des Monarchen) im Ausnahmezustand im Gegensatz zu Deutsch-

\footnotetext{
4. Stuttgart-Berlin-Köln-Mainz 1964, besonders Teil I *Belastungen der parlamentarıschen Demokratıe in Deutschland.

"Rule of Lawe in einer such wandelnden Welt (1943/44), in: Fraenkel, Reformismus und Pluralismus (FN 7), S. 258-274.
} 
land immer auf die normative Ordnung und deren Wiederherstellung bezogen ist, ein Maßnahmenstaat sich also nicht einmal theoretisch entwickeln könne. Auch hätten sich die Gerichte die Feststellung des Ausnahmezustandes stets vorbehalten. $\mathrm{Daß}$ die Prärogative in dem Augenblick verschwand, als das Bürgertum den Staat voll in seinem Besitz hatte, und es nun möglich war, den Ausnahmezustand ausschließlich in Bezug zur etablierten - nämlich bürgerlichen - Rechtsordnung zu denken, weil Rechtsordnung und Staatsgewalt den gleichen gesellschaftlichen Inhalt hatten, vernachlässigt Fraenkel. Auch verweist der kritiklose Hinweis darauf, daß Bürgerkriegsrecht eben kein Recht sei ( $₫$ Im Falle des offenen Bürgerkriegs ... stehen die unter den Bedingungen des martial law getroffenen Maßnahmen als staatliche Notwehrakte außerhalb der regulären Rechtsordnung", S. 50) und die pure Hoffnung auf die Nachprüfbarkeit durch die Gerichte auf eine »reformistische Blindheit, die nicht mehr sieht, daß dies ebenso gegen die Arbeiterklasse gewirkt hat (erwa bei der Bekämpfung des Chartismus im England des 19. Jahrhunderts) und wirken kann wie der Belagerungszustand des Nationalsozialismus ${ }^{\text {s. }}$. In gleicher Weise löst er das rationale bürgerliche Naturrecht von seinem gesellschaftlichen Hintergrund und analysiert es nur noch in der (subjektiv öffentlichen) Dimension der allgemeinen Menschen- und Bürgerrechte, nicht mehr aber in ihrer für die Entstehung und Entwicklung der kapitalistischen Gesellschaft funktionalen ("privaten«) Dimension. Daß das bürgerliche Naturrecht ebenso wie die bürgerliche Aufklärung Inhalte besaß und besitzt, die in der bürgerlichen Gesellschaft nicht zu verwirklichen sind, wird von ihm zwar im "Doppelstaat" noch gesehen; wirft er doch der Weimarer Sozialdemokratie vor, sie habe versucht, innerhalb der bürgerlichen Gesellschaft das Naturrecht zu verwirklichen - was unmöglich sei - und dies sei ihr zum Verhängnis geworden (S. 163). Gleichwohl dominiert das politische Ziel einer antifaschistischen "Einheitsfront der Naturrechtler" über solche kritischen Gedanken. Die schon eingangs erwähnte Ambivalenz der Fraenkelschen Theorieentwicklung zwischen den Formen, Institutionen und grundlegenden Legitimitätsvorstellungen des bürgerlichen Verfassungsstaates einerseits und der Erkenntnis der bürgerlichen Gesellschaft als Klassengesellschaft (und des bürgerlichen Staates als Klassenstaat) andererseits wird durch die Bedrohung vom Faschismus und die Enttäuschung an einer entweder apathischen oder kommunistischen Arbeiterbewegung fast völlig zugunsten des Verfassungsstaates entschieden.

Von hier aus ergibt sich eine konsequente Entwicklung zu jenen Schriften Fraenkels, die ihn in der Bundesrepublik als Theoretiker der repräsentativen und pluralistischen Demokratie bekannt gemacht haben. ${ }^{67}$ In der Betonung der Bedeutung der Repräsentation kommt die totalitarismustheoretische Gleichsetzung von faschistischen und kommunistischen Massenbewegungen und der Norwendigkeit von deren Abwehr zum Ausdruck ("plebiszitäre Herrschaft«, vgl. auch schon "Doppelstaat « S. 38); die Fraenkelsche Pluralismustheorie stellt im Kern die Rückkehr zur Hoffnung auf die friedliche Integration der Arbeiterklasse unter Mitarbeit der liberalen Bourgeoisie in den bürgerlich-demokratischen Verfassungsstaat und die Rekonstruktion einer ausschließlich auf Tarif- und Sozialpolitik beschränkten Gewerkschaftsbewegung dar, wie sie in der Weimarer Republik schon in der Vorstellung von der "kollektiven * oder "dialektischen « Demokratie angelegt war. Allerdings sind dabei zwei wesentliche Punkte zu beachten. Zwar hat Fraenkel erstens an der

\footnotetext{
\& Dagegen steht seine eher skeptısche Analyse des -Martial Law a Im Rahmen der Diskussion um die Notstandsgesetzgebung in: > Martzal Law a und Staatsnotstand in England und USA, in: Ernst Fraenkel, Hg., Der Staatsnotstand (Vorträge), Berlin 1965, S. I 38-164. Vgl. auch Franz Neumann, Der Funktzonswandel des Gesetzes ım Recht der bürgerlichen Gesellschaft, in: ders., Demokratsscher und autoritärer Staat, Frankfurt/Main 1967, S. 3 I-8I.

67 Vgl. hierzu das Kapitel 9 in Blanke (FN 13).
} 
Renaissance des Naturrechts « mitgewirkt, die nach 1945 auch und wesentlich dazu beitragen sollte, die bürgerliche Gesellschaft neu zu legitimieren, und in seiner Pluralismustheorie spielt der Gedanke, das "Gemeinwohl " nicht nur an den Konsens durch gesellschaftlichen Interessenausgleich, sondern auch an übergeordnete Werte ("Basiskonsens") zu binden, eine zentrale Rolle. Gleichwohl ist er mit den

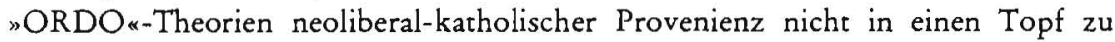
werfen. Denn das zentrale Problem des pluralistischen Gemeinwohls ist für ihn zweitens nach wie vor der gesellschaftliche Interessenkampf - auch wenn er nun glaubte, das Naturrecht könne ohne Klassenkampf verwirklicht werden. Aus diesem Grunde kann - auch wenn Fraenkel selbst dieser Meinung war - der Pluralismus nicht bruchlos als "Staatstheorie des Reformismus « ${ }^{68}$ bezeichnet werden. Bestimmte theoretische Prämissen halten sich zwar durch die Frankelsche Theorieentwicklung durch, aber im Gegensatz zu seinem Denken in der Weimarer Republik soll der "Pluralismus« eben kein Durchgangsstadium zum Sozialismus mehr sein. Das im Reformismus noch enthaltene Endziel jenseits der bestehenden Gesellschafts- und Verfassungsordnung ist aufgegeben. Die Kontinuität der Theoriebildung erstreckt sich somit nicht auf die politischen Vorstellungen. Nur unter Absehung von den Brüchen, die in der politischen Richtung Fraenkels feststellbar sind, oder wenn man sie als notwendig erachtet, kann man der Ansicht sein, der ehemalige Reformismus hätte sich zum, den bürgerlichen Staat bejahenden, Integrationismus mit pluralistischen Vorzeichen wandeln müssen.

\footnotetext{
st Vgl. hierzu Wolfgang Müller, Der Pluralismus - die Staatstheone des Reformusmus, in: Doeker/Steffanı (FN 8), S. 395-424.
} 